\title{
El escaso poder predictivo de simples curvas de Phillips en Chile
}

\author{
Pablo Pincheira Brown y Hernán Rubio Hurtado
}

RESUMEN

En este trabajo se explora la existencia, robustez y magnitud del eventual aporte que puedan tener diversas medidas de actividad en la tarea de predecir inflación en Chile, sobre la base de algunas versiones retrospectivas (backward-looking) de curvas de Phillips estimadas tanto con datos revisados como con datos en tiempo real. Los principales resultados confirman los hallazgos de la literatura reciente a nivel internacional: el aporte predictivo de las medidas de actividad aquí consideradas es episódico, inestable y de magnitud moderada. Este precario aporte predictivo es robusto a la utilización de datos definitivos y en tiempo real. Santiago, Chile. pablo.pincheira@uai.cl

Hernán Rubio Hurtado es analista económico en la Gerencia de Análisis Macroeconómico del Banco Central de Chile.hrubio@bcentral.cl 


\section{I}

\section{Introducción}

En artículos recientes sobre predicción de la inflación en los Estados Unidos de América sobre la base de curvas de Phillips se aprecia una capacidad predictiva algo limitada, que ha sido denominada por Stock y Watson (2008) como "episódica". En otras palabras, las curvas de Phillips —entendidas como modelos para predecir inflación a partir de una o más variables de actividad - tendrían capacidad predictiva solo en algunos períodos específicos, mientras que en otros esta capacidad prácticamente desaparecería o no sería superior a la de algunos competidores simples. Este tipo de resultados, desde luego llamativos e inquietantes, han sido reportados no solo por Stock y Watson (2008), sino además por Rossi y Sekhposyan (2010), y Clark y McCracken (2006), e implícitamente también por Ciccarelli y Mojon (2010), entre otros.

Las curvas de Phillips, en sus distintas versiones, han estado presentes en el análisis económico desde hace muchos años. Sin embargo, los resultados de Stock y Watson (2008); Rossi y Sekhposyan (2010), y Clark y McCracken (2006) ponen en tela de juicio el uso predictivo que se le puede dar a este tipo de relaciones en la literatura económica.

La discusión en tal sentido está llena de sutilezas que pueden ser importantes a la hora de juzgar la utilidad predictiva de una curva de Phillips. En primer lugar, la variedad existente de curvas de Phillips hace prácticamente imposible la evaluación de todas ellas en un solo trabajo académico. En segundo lugar, dichas curvas surgen como una relación contemporánea entre variables de actividad e inflación, lo que en estricto rigor las convierte en modelos de consistencia y no en modelos de proyección. Este es el caso, por ejemplo, de la curva de Phillips neokeynesiana, que en términos

Los autores agradecen los acertados comentarios de un árbitro anónimo, como también a Carlos Medel y Ramón Cornejo por su valiosa asistencia y a María Pilar Pozo por facilitarnos parte de la base en tiempo real del Indicador Mensual de Actividad Económica (IMACEC). Nuestro trabajo también se ha beneficiado con las opiniones expresadas en los talleres de Conducción de Política Monetaria y de Dinámica de Precios y Salarios del Banco Central de Chile, así como en los Seminarios de Economía del Banco Central de la República Argentina. Valiosos comentarios de Luis Felipe Céspedes, Claudio Soto y Pablo García también han sido incorporados a este trabajo. Las opiniones vertidas en este artículo no necesariamente representan las opiniones del Banco Central de Chile o la de sus Consejeros. generales plantea una relación contemporánea entre inflación, costos marginales y expectativas de inflación (véase, por ejemplo, Céspedes, Ochoa y Soto, 2005). En principio, no es claro que el modesto desempeño predictivo de las curvas de Phillips necesariamente implique también una débil relación contemporánea entre medidas de actividad e inflación.

Para entender la evidencia de la precaria utilidad predictiva antes mencionada, se han planteado algunas hipótesis. En particular, para el caso de los Estados Unidos de América, se ha dicho que la falta de capacidad de ciertas medidas de actividad para predecir inflación no obedecería necesariamente a una ausencia de relación entre medidas usuales de actividad e inflación futura, sino a una relación débil entre ambas variables, que de ser lineal podría estar vinculada a un parámetro pequeño y probablemente inestable ${ }^{1}$. Esta visión es consistente con una serie de trabajos que han reportado cierta inestabilidad en los parámetros de modelos de inflación para países tan diversos como el Canadá (Hostland, 1995); Colombia (Melo y Misas, 1997); Estados Unidos de América (Russell y Chowdhury, 2013) y Venezuela (República Bolivariana de) (Pagliacci y Barráez, 2010).

Parece relevante, por lo tanto, explorar esta hipótesis para la relación entre medidas usuales de actividad e inflación futura en Chile. Así, en este trabajo se analiza si algunas medidas tradicionales de actividad tienen capacidad para aportar en la tarea predictiva de la inflación en el país. En el caso de que la respuesta sea afirmativa, se estudiará la estabilidad de esta capacidad predictiva.

En el objetivo aquí planteado impera un sentido fundamentalmente pragmático. El interés último de la investigación consiste en determinar si las medidas de actividad acá analizadas pueden iluminar una toma de decisión de política económica, sobre la base de un adecuado pronóstico de inflación. Para ello se utiliza una base en tiempo real del Índice Mensual de Actividad Económica (IMACEC), que entrega en cada momento del tiempo $t$ la serie histórica de dicho Índice que se encontraba disponible en ese instante en los Boletines Mensuales del Banco Central de Chile (BCCH). Este

\footnotetext{
${ }^{1}$ Esta hipótesis fue planteada por Michael McCracken en las Joint Statistical Meetings 2009, celebradas en Washington D.C., en agosto de 2009.
} 
punto es muy importante, sobre todo porque las cifras de actividad generalmente pasan por varias rondas de revisiones hasta convertirse en datos definitivos. Estas rondas de revisiones pueden tardar años y, tal como se muestra en este artículo, pueden conducir a importantes modificaciones con respecto a las primeras señales de actividad publicadas. Una evaluación de la utilidad predictiva de medidas de actividad sobre la base de cifras revisadas del IMACEC, podría en principio ser muy poco útil para juzgar el aporte de estas variables en la toma de decisiones económicas. Si la diferencia entre las primeras cifras publicadas y las definitivas fuera considerable, cualquier análisis de esta naturaleza efectuado con cifras definitivas estaría contaminado, porque contendría datos que en el momento de la toma de decisiones no pertenecían al conjunto de información disponible. Por esta razón, en este artículo se le asigna un papel importante a las estimaciones en tiempo real, aunque paralelamente se realizan estimaciones con cifras definitivas, a objeto de evaluar las potenciales diferencias que se puedan detectar entre el análisis con cifras revisadas y el análisis en tiempo real.

Los principales resultados obtenidos coinciden con los mostrados por Stock y Watson (2008); Rossi y Sekhposyan (2010), y Clark y McCracken (2006) para los Estados Unidos de América: la evidencia de predictibilidad en Chile es episódica, inestable y, en general, el coeficiente que acompaña a las distintas medidas de actividad es de magnitud moderada. Estos hallazgos pueden explicar en parte algunos de los resultados obtenidos en el ejercicio fuera de muestra que también se llevó a cabo. En este ejercicio, se advierte que el aporte predictivo de las medidas de actividad que se analizaron es mínimo o inexistente con respecto al aporte de los rezagos de la inflación. De estos resultados empíricos se concluye que, si bien las medidas de actividad aquí utilizadas tienen cierta capacidad para predecir inflación, esta capacidad es inestable y modesta en relación con el aporte de componentes tendenciales y estacionales de la inflación en Chile.

Es importante enfatizar que los resultados del presente estudio provienen de un riguroso y básico análisis econométrico en torno de cuatro simples versiones de curvas de Phillips de tipo backward-looking, en que la variable de actividad utilizada ingresa en cada ecuación sin más rezagos que el último dato de actividad disponible ${ }^{2}$. En principio, no parece directo generalizar estos resultados con respecto a otras versiones de curvas de Phillips que incorporen términos adelantados forward-looking, otras variables de actividad, rezagos adicionales de ellas o ambos. Desde este punto de vista, sería interesante estudiar en el futuro qué tan extrapolables son los resultados a este tipo de especificaciones. La elección de curvas de Phillips de tipo backward-looking en este trabajo obedece a la extensa literatura que las ha estudiado en el último tiempo, que aparece citada en la siguiente sección, y al hecho de que una instrumentación usual de términos forward-looking consiste simplemente en añadir rezagos de la variable en cuestión, expresión que finalmente se parece bastante a una especificación backward-looking. Por último, es importante aclarar la razón por la cual en este caso solo se incorporó el último dato de medida de actividad disponible sin incluir rezagos. Esto se hizo debido a la importancia que parece tener en el debate el estado actual de la actividad económica de un país por sobre la evolución de esa actividad. En particular, se toma como base el hecho de que la curva de Phillips utilizada por el denominado modelo estructural de proyección (MEP) del вCCH (Banco Central de Chile, 2003) - así como una especificación tradicional de una regla de Taylor - incluye solo al término contemporáneo de la brecha del producto y no a rezagos adicionales (Taylor, 1993).

El resto del artículo se organiza de la siguiente manera. En la sección II se presenta una pequeña revisión de la literatura reciente respecto de la predictibilidad de inflación sobre la base de curvas de Phillips. En la sección III se describe la metodología adoptada en este trabajo. En la sección IV se muestran los resultados, en la sección $\mathrm{V}$ se lleva a cabo un somero análisis de robustez, mientras que en la sección VI se entregan las principales conclusiones derivadas de este estudio.

\footnotetext{
2 También en el presente estudio se ha realizado un somero análisis de robustez inspirado en los trabajos de Hostland (1995) y Melo y Misas (1997), así como en el de Pagliacci y Barráez (2010), en el sentido de ampliar las especificaciones para permitir cambios de régimen o incorporar como variable adicional de control la tasa de variación anual del tipo de cambio.
} 


\section{II}

\section{Revisión de la literatura}

Desde hace largos años, diversos autores detectaron empíricamente relaciones entre actividad económica e inflación, que posteriormente se han popularizado bajo el nombre de curvas de Phillips, en alusión al trabajo de Phillips (1958). Tanto este autor como Fisher (1926) y Samuelson y Solow (1960) documentan la existencia de una relación empírica inversa entre alguna medida de inflación y la tasa de desempleo. Posteriormente, son incontables los artículos en que se debate y argumenta en favor y en contra de la existencia, estabilidad y utilidad, o ambas de este tipo de relaciones. Para el lector interesado se recomienda la reseña de Atkeson y Ohanian (2001), que entrega una breve revisión histórica de la literatura al respecto. De modo similar, también es relevante revisar el artículo de Stock y Watson (2008), quienes hacen un resumen de la literatura en que se evalúan predicciones de inflación con metodología pseudo-fuera de muestra para los Estados Unidos de América desde el año 1993.

Si bien sería demasiado ambicioso intentar cubrir en algunos breves párrafos toda la rica y vasta literatura en que se analizan y utilizan diversas medidas de actividad como fundamento de la inflación, parece relevante dedicar algunas líneas a aquellas contribuciones más o menos recientes en que se procura específicamente utilizar curvas de Phillips o medidas de actividad con el fin de predecir inflación.

En relación con la revisión bibliográfica aquí comenzada, es interesante destacar una suerte de contradicción entre diversos artículos escritos en la última década. En efecto, para dar un ejemplo de la oscilación de opiniones se aludirá primeramente a los artículos de Stock y Watson (1999 y 2008). En el primero de sus artículos, los autores señalan que dentro de los métodos utilizados para predecir inflación, las curvas de Phillips son consideradas estables y confiables en la generación de pronósticos. De hecho, en este artículo, Stock y Watson (1999) dedican parte de su esfuerzo a evaluar la estabilidad de una determinada curva de Phillips, que incluye desempleo y rezagos de inflación como predictores. Si bien estos autores detectan cierta inestabilidad en esta ecuación, ella es atribuida sobre todo a los coeficientes asociados a los rezagos de la inflación, mientras que los coeficientes relacionados con medidas de actividad económica son hallados relativamente estables.
Paralelamente, documentan que medidas de actividad, distintas a desempleo, pueden generar predicciones más precisas que aquellas que solo utilizan variables vinculadas a empleo ${ }^{3}$. Finalmente, los autores concluyen que las curvas de Phillips son instrumentos útiles para predecir inflación. Diez años más tarde la historia parece ser otra, puesto que los mismos autores en el año 2008 escriben un artículo en que señalan que pronósticos basados en curvas de Phillips tienen un comportamiento "episódico", esto es, que en algunos períodos son superiores a un buen marco de referencia univariado (benchmark), pero que en algunos otros son simplemente superados por estos buenos benchmarks univariados.

Si bien los resultados mostrados por Stock y Watson en este período de 10 años no están en franca contradicción, de alguna manera dejan ver una atenuación en el entusiasmo inicial con respecto a la utilidad de las curvas de Phillips como métodos de predicción.

Un resultado algo más drástico que el reciente de Stock y Watson es entregado por Atkeson y Ohanian (2001), quienes notan que varias especificaciones de curvas de Phillips son incapaces de predecir la inflación de los Estados Unidos de América un año hacia adelante, de manera más precisa que un simple camino aleatorio. Este resultado rememora amargamente el devastador artículo de Meese y Rogoff (1983) en el terreno de la literatura de predicción de tipos de cambio.

Siguiendo con este paralelo respecto de la literatura de predicción de tipos de cambio, Clark y McCracken (2006) señalan que existiría evidencia en favor de la capacidad predictiva de las curvas de Phillips cuando esta predictibilidad es evaluada en ejercicios dentro de muestra, y evidencia mixta de esta predictibilidad cuando es evaluada mediante ejercicios fuera de muestra. En un intento por reconciliar estos dos resultados un tanto contradictorios, estos autores exploran dos posibles explicaciones: la inestabilidad en los parámetros de la curva de Phillips y la potencia de las pruebas fuera de muestra. Los autores concluyen que es la falta de poder de las pruebas fuera de muestra, en relación con las pruebas dentro de muestra, lo que podría explicar

\footnotetext{
${ }^{3}$ El período de análisis va desde enero de 1959 a septiembre de 1997 en frecuencia mensual.
} 
tales resultados. Si bien esta falta de poder se podría ver amplificada por una supuesta inestabilidad en los parámetros de la curva de Phillips, ellos mencionan una serie de artículos sugiriendo más bien estabilidad que inestabilidad en la curva de Phillips (véanse, por ejemplo, Stock y Watson, 1999; Rudebusch y Svensson, 1999; Estrella y Fuhrer, 2003).

Otro resultado interesante $-\mathrm{y}$ que de alguna $\mathrm{u}$ otra forma representa también una oscilación en la literatura- es la contribución de Rossi y Sekhposyan (2010), quienes encuentran que la capacidad predictiva de las curvas de Phillips desapareció al comienzo del período denominado como la Gran Moderación, en lo que es otro resultado en dirección opuesta a los de Stock y Watson (1999); Rudebusch y Svensson (1999), y Estrella y Fuhrer (2003), porque da cuenta de una inestabilidad predictiva de las curvas de Phillips que, de acuerdo con Clark y McCracken (2006), no es reportada en estos últimos artículos. De igual modo, y como ya se ha señalado en la Introducción, para los casos de la inflación de los Estados Unidos de América, el Canadá, Colombia y Venezuela (República Bolivariana de), también se encuentra evidencia de inestabilidad en los parámetros de algunas especificaciones estimadas por Hostland (1995); Melo y Misas (1997); Russell y Chowdhury (2013), y Pagliacci y Barráez (2010).

Para el caso de Chile, al parecer existen pocos trabajos en que se estudie la capacidad predictiva de alguna variante de curva de Phillips sobre la inflación. La revisión de la literatura efectuada en el presente estudio condujo a cuatro trabajos: Nadal de Simone (2001); Aguirre y Céspedes (2004); Fuentes, Gredig y Larraín (2008), y Morandé y Tejada (2008). En el primer trabajo, Nadal de Simone (2001) estima una curva de Phillips con parámetros variables para Chile y encuentra, en un análisis dentro de muestra, que todos los coeficientes son significativos ${ }^{4}$. No obstante, es muy llamativa la evolución del coeficiente asociado a la brecha de producto que presenta este autor. En primer lugar, el coeficiente parte tomando valores negativos al inicio de la década de 1990, para luego alcanzar un máximo positivo alrededor de 1995 y comenzar una declinación rápida hacia fines de dicha década, alcanzando un valor cercano a cero. Este patrón de "U" invertida es muy llamativo, puesto que trasluce una trayectoria persistente que abarca valores

\footnotetext{
${ }^{4}$ Nadal de Simone (2001) también realiza un análisis fuera de muestra, pero solo considerando cuatro pronósticos de inflación. Debido al pequeño número de observaciones, el presente trabajo se centra en sus conclusiones provenientes del análisis dentro de muestra.
}

positivos, negativos y que finalmente se acerca a cero, indicando que si la brecha tuvo alguna vez relevancia en predecir la inflación, esta se fue perdiendo hacia fines del período muestral.

Otro trabajo muy interesante es el de Aguirre y Céspedes (2004). Estos autores evidencian que una curva de Phillips, aumentada con factores dinámicos de acuerdo con la metodología fuera de muestra de Stock y Watson (1998), mejora la capacidad predictiva de una curva de Phillips tradicional para horizontes de 6, 9 y 12 meses. Este modelo aumentado también supera a un benchmark univariado en horizontes de 9 y 12 meses. Por otra parte, Fuentes, Gredig y Larraín (2008) evalúan la capacidad predictiva fuera de muestra de varias curvas de Phillips en lo que ellos llaman un ejercicio predictivo "casi" en tiempo real. Este ejercicio se diferencia de uno en tiempo real, entre otras cosas, porque utiliza cifras revisadas de producto interno bruto (PIB) y no realiza una desestacionalización en tiempo real. Con estas consideraciones, los autores encuentran que las medidas de brecha de producto tienen capacidad predictiva para la inflación en horizontes de 3 a 4 trimestres. Finalmente, aunque sin objetivos predictivos, Morandé y Tejada (2008) también estiman una curva de Phillips con parámetros variables en el tiempo. Además, ellos descomponen la evolución de los parámetros de esta curva en períodos de alta y baja volatilidad. Sus resultados denotan una marcada oscilación del parámetro de la brecha asociado al estado de alta inestabilidad de la economía. Igualmente, el parámetro parece presentar una tendencia menguante a lo largo del tiempo, al menos en períodos de estabilidad, lo que indicaría una decreciente capacidad de la brecha de producto para predecir inflación.

Se aprecia entonces que la evidencia de predictibilidad sobre la base de curvas de Phillips para Chile es heterogénea. Tanto el trabajo de Aguirre y Céspedes (2004) como el de Fuentes, Gredig y Larraín (2008) muestran capacidad predictiva, pero los trabajos de Nadal de Simone (2001) y Morandé y Tejada (2008) dan cuenta de un parámetro de brecha inestable, lo que dificultaría el potencial predictivo de las curvas de Phillips.

Es importante destacar que en la mayoría de estos artículos se trabaja con cifras revisadas que pueden distar bastante de las cifras en tiempo real. Chumacero y Gallego (2002) muestran que la diferencia entre series revisadas del IMACEC y sus primeras señales puede ser notable. Más recientemente, Morandé y Tejada (2008) denotan importantes discrepancias entre diversas estimaciones de brecha obtenidas en tiempo real y con cifras revisadas. De hecho, estos autores señalan que en la literatura ya se ha sugerido seguir reglas de comportamiento de política 
monetaria basadas en variables que sean inmunes a este tipo de incertidumbre.

De la revisión de la literatura queda claro que aún está pendiente un análisis de predictibilidad en tiempo real usando curvas de Phillips en Chile. Este análisis

\section{III}

\section{Metodología}

\section{Especificaciones econométricas}

En este trabajo, el objetivo fundamental es evaluar la capacidad predictiva que tienen ciertas medidas de actividad respecto de la inflación futura en Chile. Para ello se adoptan cuatro simples modelos lineales, algunos de ellos muy similares a los utilizados por Aguirre y Céspedes (2004) y Fuentes, Gredig y Larraín (2008), o los modelos de inflación de Stock y Watson (2008). De esta forma, se considerará la siguiente familia de modelos:

$$
\begin{gathered}
\pi_{t+h}=\delta_{1} \bar{\pi}_{t}+\alpha_{1}+\gamma_{1}\left(Y_{t-1}-Y_{t-1}^{*}\right) \\
+\sum_{i=0}^{n} \varphi_{1, i} \pi_{t-i}+\varepsilon_{1, t+h} \\
\pi_{t+h}=\delta_{2} \bar{\pi}_{t}+\alpha_{2}+\gamma_{2} 100\left(\ln \left[Y_{t-1}\right]-\ln \left[Y_{t-1}^{*}\right]\right) \\
+\sum_{i=0}^{n} \varphi_{2, i} \pi_{t-i}+\varepsilon_{2, t+h} \\
\pi_{t+h}=\delta_{3} \bar{\pi}_{t}+\alpha_{3}+\gamma_{3} 100\left(\ln \left[Y_{t-1}\right]-\ln \left[Y_{t-13}\right]\right) \\
+\sum_{i=0}^{n} \varphi_{3, i} \pi_{t-i}+\varepsilon_{3, t+h} \\
\pi_{t+h}-\pi_{t}=\delta_{4} \bar{\pi}_{t}+\alpha_{4}+\gamma_{4} 100\left(\ln \left[Y_{t-1}\right]-\ln \left[Y_{t-1}^{*}\right]\right) \\
+\sum_{i=0}^{n} \varphi_{4, i} \pi_{t-i}+\varepsilon_{4, t+h}
\end{gathered}
$$

donde:

$$
\pi_{t+h}=100\left[\ln \left(P_{t+h}\right)-\ln \left(P_{t+h-12}\right)\right]
$$

denota la aproximación logarítmica de la inflación acumulada en 12 meses hasta el mes $t+h$. Esta inflación se mide de acuerdo con el índice de precios al consumidor (IPC). permitiría evaluar la real capacidad de estas curvas para proveer de proyecciones confiables de inflación a los agentes tomadores de decisiones. Precisamente esto es lo que se lleva a cabo en las siguientes secciones de este artículo.
Por otra parte, $Y_{t-1}$ denota al IMACEC desestacionalizado mediante el método x12-ARIMA. $Y_{t-1}$ es una medida de actividad económica disponible en el momento $t$-1. Cabe notar que este índice se publica con un mes de rezago con respecto a la inflación. Así, en diciembre de 2009 - por ejemplo- se publica la inflación de noviembre de 2009 y el IMACEC de octubre del mismo año. A ello se debe que en el lado derecho en todas las ecuaciones aparezca la inflación en el instante $t$ y la medida de actividad en el instante $t-1$. En la sección de resultados de este artículo se mostrarán gráficamente algunas estimaciones de los parámetros que acompañan a la variable de actividad en las ecuaciones (1) a (4). Esto se hace estimando (1) a (4) tanto con cifras definitivas del IMACEC, como con series en tiempo real, que corresponden a las series de IMACEC que en cada mes reporta el Boletín Mensual del Banco Central de Chile (вССн).

Además, en las ecuaciones aparece $\overline{\pi_{t}}$, que es definida como la meta de inflación anunciada por el ВСCH. En la medida que exista perfecta credibilidad, este término también puede ser entendido como una manera de aproximar las expectativas de inflación ${ }^{5}$.

La variable $Y_{t-1}^{*}$ representa la tendencia del IMACEC desestacionalizado en el instante $t-1$. Esta tendencia es obtenida mediante la aplicación del filtro de HodrickPrescott.

Finalmente, las variables $\varepsilon_{i, t+h}$ representan perturbaciones no correlacionadas con la información disponible en $t$.

Dependiendo del número de rezagos de la inflación que se considere en cada ecuación, y de la inclusión o exclusión de la variable $\overline{\pi_{t}}$, se tendrá un total de $2(n+1)$ especificaciones relacionadas con cada ecuación.

\footnotetext{
5 En estricto rigor, antes de que en Chile se alcanzara una meta de inflación estable y constante igual a 3\%, la meta de inflación era variable, y en cierto período muestral correspondía a una meta a diciembre de cada año y no a la inflación acumulada en los últimos 12 meses.
} 
En general, en este estudio siempre se trabajará con al menos el término contemporáneo de inflación en el lado derecho, por lo que las posibles especificaciones se reducen a $2 n$. El objetivo se centra en determinar el tamaño, la estabilidad y la significancia estadística de los cuatro parámetros $\gamma_{i}, i=1,2,3,4$. Para obtener estimaciones robustas de cada uno de estos parámetros, es decir, estimaciones que no dependan de cada una de las $2 n$ especificaciones posibles para cada ecuación, se aplicará la tradicional metodología de promedios bayesianos (BMA, por sus siglas en inglés) descrita, entre otros, por Brock y Durlauf (2001) y que también puede encontrarse resumida en el anexo $\mathrm{C}$ del artículo de Pincheira y Calani (2010).

\section{Estimación, simultaneidad y endogeneidad}

Tal como se ha señalado anteriormente, en este artículo se utiliza la expresión "curvas de Phillips" para denotar una relación genérica entre inflación y una o más variables de actividad. Estas relaciones aparecen en la literatura económica fundamentalmente con dos funciones $\mathrm{u}$ objetivos. En primer lugar, ecuaciones que establecen una relación entre inflación y actividad típicamente forman parte de un conjunto de ecuaciones simultáneas en modelos de equilibrio general, que intentan describir la mecánica de una serie de variables macroeconómicas en su conjunto. Un ejemplo de esto es el modelo MEP del вссн (Banco Central de Chile, 2003), en el que se utiliza una expresión muy similar a las empleadas en el presente artículo, aunque extendida para incluir además un término de inflación importada. Un ejemplo algo distinto se encuentra en Yeh (2009), quien más que elaborar un modelo de equilibrio general para la economía, intenta determinar la relación causal que existe entre crecimiento e inflación y, por otra parte, entre inflación y crecimiento. Esto lo lleva a plantear un modelo de dos ecuaciones simultáneas, en que tanto el crecimiento como la inflación son variables endógenas. En este caso, y en general en sistemas de ecuaciones simultáneas, Yeh (2009), así como Hansen (2014), muestran que el estimador de mínimos cuadrados ordinarios (MCO) de cada ecuación genera estimadores inconsistentes de los parámetros estructurales del modelo. Para resolver este inconveniente es necesario recurrir a información adicional a la contenida en las propias ecuaciones, a fin de obtener una estimación consistente. Con ese propósito, es tradicional recurrir a variables instrumentales o a estrategias de identificación por heterocedasticidad. Aplicaciones o variaciones interesantes de estas metodologías se encuentran en Russell y Chowdhury
(2013) y en García-Solanes y Torrejón-Flores (2012), así como en el ya citado artículo de Yeh (2009), por mencionar solo algunos trabajos.

En segundo lugar, en otra parte de la literatura se ocupa una relación entre inflación y actividad con fines predictivos. Tal es el caso del presente trabajo y de los ya mencionados artículos de Stock y Watson (2008); Rossi y Sekhposyan (2010); Clark y McCracken (2006), y Ciccarelli y Mojon (2010).

Cuando el objetivo es de carácter predictivo, es usual usar modelos uniecuacionales, multi o univariados, basados en los siguientes resultados teóricos:

i) El mejor predictor bajo pérdida cuadrática para una variable $Y_{t+h}$ basado en la información disponible en un vector de variables $X_{t}$ está dado por la esperanza condicional de $Y_{t+h}$, dado $X_{t}$ es decir $E\left(Y_{t+h} \mid X_{t}\right)$ ) (para la demostración, véase Hansen, 2014).

ii) El mejor predictor lineal de una variable $Y_{t+h}$ basado en la información disponible en el vector de variables $X_{t}$ está dado por $X_{t}^{T} \beta^{*}$, en que $\beta^{*}$ está definido como

$$
\beta^{*}=\left[E\left(X_{t} X_{t}^{T}\right)^{-1}\right] E\left(X_{t} Y_{t+h}\right)
$$

y se denomina mejor predictor lineal bajo pérdida cuadrática para $Y_{t+h}$, basado en la información disponible en un vector de variables $X_{t}$ (para la demostración véase Hansen, 2014).

iii) El estimador de MCo entre $Y_{t+h}$ y el vector de variables $X_{t}$ estima en forma consistente al mejor predictor lineal definido en el punto anterior (véase Hamilton, 1994).

Los tres resultados antes señalados son la base sobre la que se fundamenta la construcción y estimación de muchos modelos predictivos. De estos resultados se desprende que el tradicional problema de endogeneidad, que surge a la hora de estimar muchas relaciones en economía, no existe en el ámbito predictivo cuando el vector de parámetros que se desea estimar es el mejor predictor lineal $\beta^{*}$, lo que es el objetivo del presente estudio, ya que el estimador de MCO provee una estimación consistente. De esta manera, en este trabajo se procede a estimar las cuatro especificaciones econométricas con el método de MCO, y se interpreta este estimador como una aproximación al mejor predictor lineal ${ }^{6}$.

\footnotetext{
${ }^{6}$ Es interesante señalar, además, que en caso de que las perturbaciones de los modelos sean normales, se tendrá que el mejor predictor adquiere una forma lineal, por lo que en este caso particular el mejor predictor coincide con el mejor predictor lineal.
} 


\section{IV}

\section{Resultados empíricos}

\section{IMACEC definitivo y en tiempo real}

Las cifras de actividad, como el PIB y el IMACEC, sufren varias rondas de revisiones desde el momento en que se dan a conocer por primera vez. Así, en general, se debiesen esperar discrepancias entre la "primera señal" y la cifra final de una de estas variables. El proceso total hasta llegar a la cifra final (que es la que no se someterá a posteriores revisiones) puede tardar varios años, lo que potencialmente podría ser relevante a la hora de tomar decisiones de política económica. En efecto, si las primeras señales del PIB, por ejemplo, subestimaran significativamente a la cifra final, las decisiones de los agentes económicos podrían no ser óptimas, debido a que estarían siendo alimentadas con una primera señal sesgada. En Chile ya hay evidencia de que las diferencias entre las cifras definitivas y preliminares de actividad no han sido despreciables. Tal como se ha señalado anteriormente, Chumacero y Gallego (2002) evidencian que la diferencia entre series revisadas del IMACEC y sus primeras señales puede ser notable. Más recientemente, Morandé y Tejada (2008) dan cuenta de importantes discrepancias entre diversas estimaciones de brecha de producto obtenidas en tiempo real y con cifras revisadas. Finalmente, en Pincheira (2010) se entrega un cuadro con cifras cuasifinales y preliminares para el crecimiento del PIB anual de Chile. En este cuadro se aprecia que las primeras señales han subestimado en forma importante al PIB cuasifinal, aunque esta subestimación ha decaído notablemente en el tiempo ${ }^{7}$.

En esta subsección se hará algo distinto a lo que se ha hecho en la literatura reciente para Chile. Si bien parece importante cuantificar las diferencias entre cifras finales y preliminares como lo hacen Chumacero y Gallego (2002) y en algún sentido Pincheira (2010), se trabajará con el supuesto de que los agentes económicos realizan sus análisis considerando las series de actividad más actualizadas disponibles en cada momento del tiempo. Si se piensa en la serie del IMACEC más actualizada

\footnotetext{
${ }^{7}$ El crecimiento del PIB cuasifinal corresponde a la última cifra de crecimiento que se publica en una determinada base. Muchas veces, la cifra cuasifinal de crecimiento del PIB coincide con la cifra final que ya no sufrirá ningún tipo de revisión en el futuro. Pedersen (2013) es otro reciente trabajo en que se utiliza una base del IMACEC en tiempo real.
}

disponible en diciembre de 2009, por ejemplo, es muy probable que el último dato sea una primera señal, pero también es probable que el penúltimo dato de la serie esté en su segunda revisión, y que la cifra de diciembre de 2008 esté en una tercera o cuarta revisión. De la misma manera, es probable que el dato de diciembre de 2000 corresponda a una cifra final. Así, los agentes económicos se encuentran con series de tiempo heterogéneas, compuestas por una combinación de cifras finales y cifras en distintas rondas de revisión. Una pregunta relevante es si esta heterogeneidad inducirá algún tipo de ruido o sesgo en las variables que se deseen estimar. Morandé y Tejada (2008) responden afirmativamente a esta pregunta para el cálculo de la brecha de producto. En este trabajo se intentará evaluar diferencias en la capacidad que distintas medidas de actividad tengan para predecir inflación. También se procurará considerar las potenciales diferencias que puedan existir en los pronósticos de inflación propiamente tales. No obstante, previo a esta directa evaluación de las preguntas de interés, es aconsejable evaluar gráficamente si las diferencias entre las series en tiempo real y las series definitivas son de magnitud importante. En los gráficos 1 a 3 se muestran secuencias de series de tiempo que corresponden a las diferencias entre las series disponibles en tiempo real y las series con datos definitivos. Los paneles de los gráficos difieren en el año base que es considerado para el cálculo de las cifras de actividad. Cada panel dentro de cada gráfico representa la diferencia entre el IMACEC definitivo y el que estaba publicado en el Boletín Mensual del вCCH en marzo de cada año. Estos resultados se presentan para una submuestra del período comprendido entre 1997 y 2009. Cabe notar que en los paneles se aprecia una zona sombreada indicativa de que en los valores de ese período se incluyen datos no definitivos. En estas zonas se calcula la diferencia entre la última señal disponible y la señal en tiempo real correspondiente.

En el gráfico 1 se analizan las curvas solo hasta el mes de diciembre de 1995. Esto es porque a partir de enero de 2006 no existen cifras definitivas del IMACEC que utilicen como base el año 1986, toda vez que rondas posteriores de revisiones continuaron efectuándose con la base de 1996. Como no se desea comparar cifras en distintas bases, se considera preferible centrarse en los 
datos disponibles solo hasta diciembre de 1995. En el primer panel (correspondiente al Boletín Mensual de marzo de 1996), se muestra una revisión importante entre las cifras en tiempo real y las definitivas. Coherentes con el resultado de Pincheira (2010), las cifras en tiempo real subestiman significativamente a las cifras definitivas, y mientras menos rondas de revisiones tengan, mayor es la subestimación. Este mismo patrón se observa en el segundo panel del gráfico 1. No obstante, en los dos siguientes paneles se advierte que prácticamente no hubo revisiones en las publicaciones de marzo de 1998 en adelante para las cifras anteriores a enero de 1996. Esto indica que a marzo de 1998, las cifras de diciembre de 1995 y anteriores ya eran prácticamente definitivas.

En el gráfico 2 aparece un escenario muy distinto al del gráfico 1 . Conviene recordar que en el gráfico 2 se comparan series con base de 1996. Por las mismas razones esbozadas en el párrafo anterior, solo se compararán las cifras hasta diciembre de 2002, fecha hasta la que existen cifras definitivas del IMACEC sobre la base de 1996. Los cuatro paneles del gráfico 2 son muy distintos a los del gráfico 1. En primer lugar, durante los 5 años de evolución que abarca el gráfico 2, las revisiones fueron permanentes, pues todos los paneles muestran discrepancias entre las series definitivas y en tiempo real. Segundo, el patrón de revisiones de cada panel es distinto al que se advierte en el primer panel del gráfico 1 . Ahora no se aprecia una tendencia creciente en los paneles, ni un sesgo de subestimación del IMACEC definitivo tan marcado como en el gráfico 1. Es notable, además, que las revisiones que se muestran en el gráfico 2 son de tamaño mediano y presentan un cierto patrón estacional.

Evolución de la diferencia entre el IMACEC final y en tiempo real (IF - ITR) (Índice año base $1986=100$ )

Boletín Mensual de marzo 1996

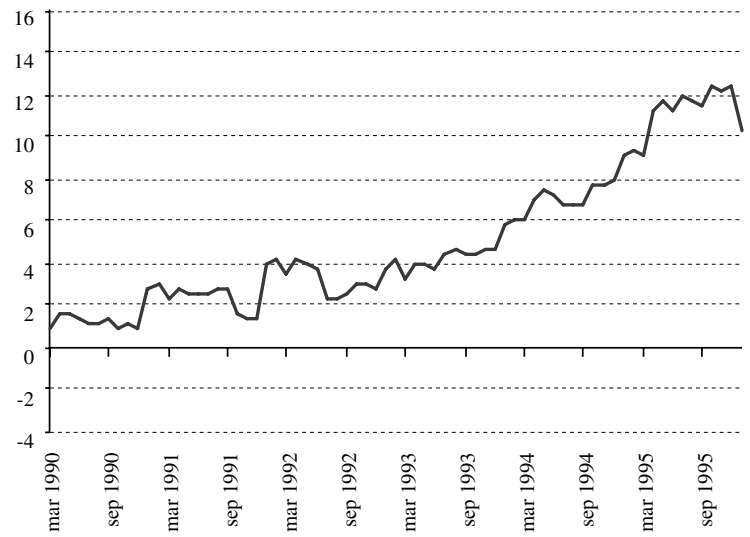

Boletín Mensual de marzo 1998

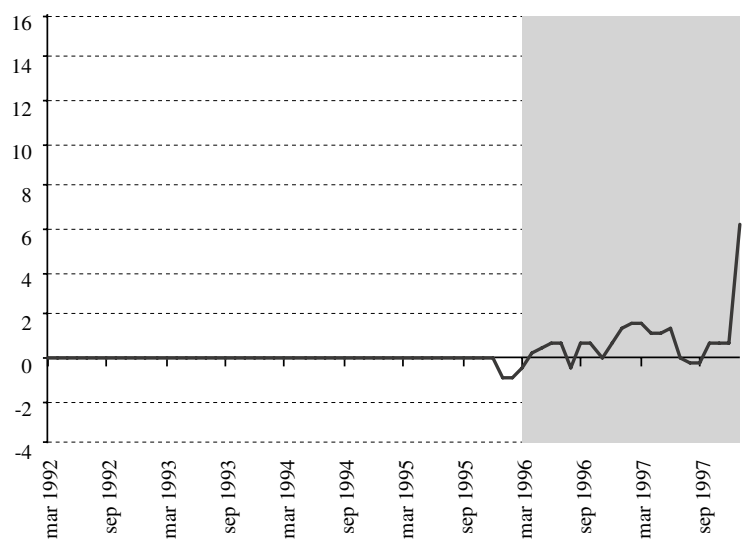

Boletín Mensual de marzo 1997

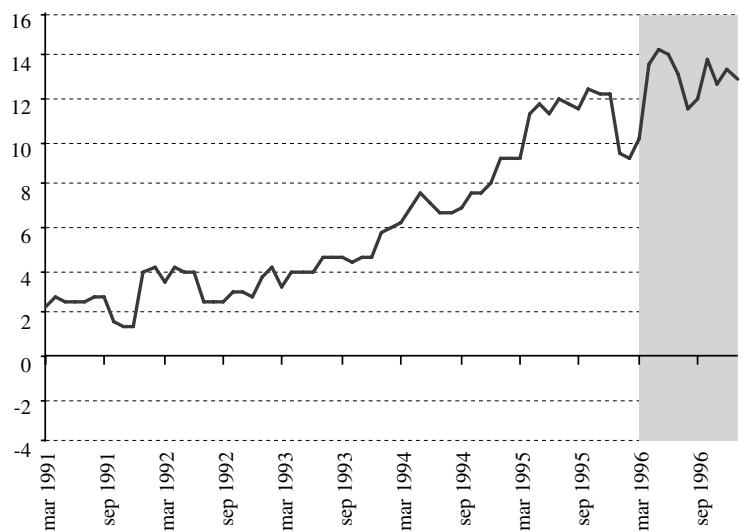

Boletín Mensual de marzo 1999

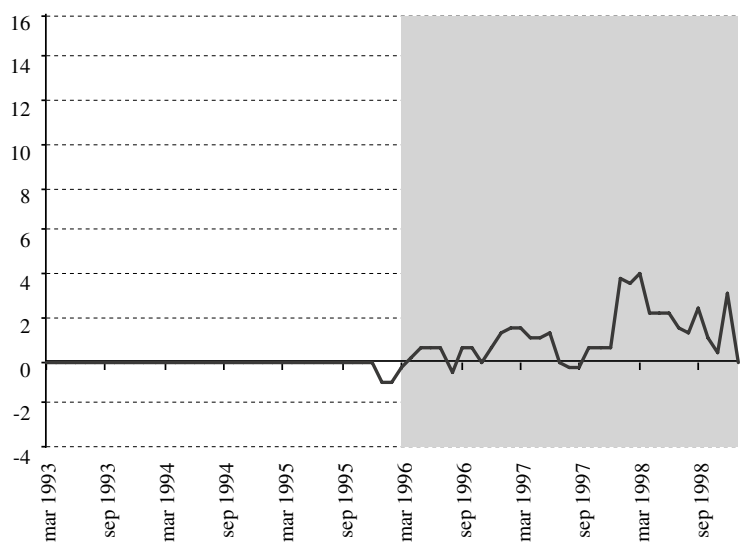

Fuente: Banco Central de Chile. 
Evolución de la diferencia entre el IMACEC final y en tiempo real (IF - ITR) (Índice año base 1996=100)

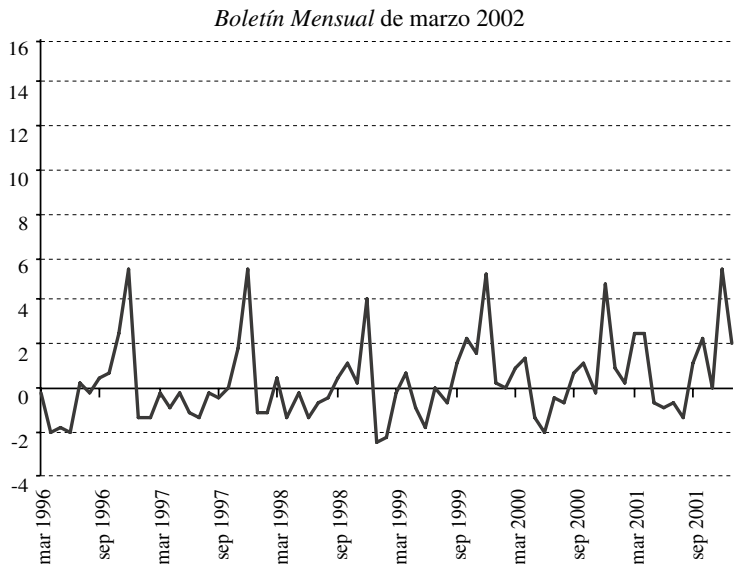

Boletín Mensual de marzo 2004

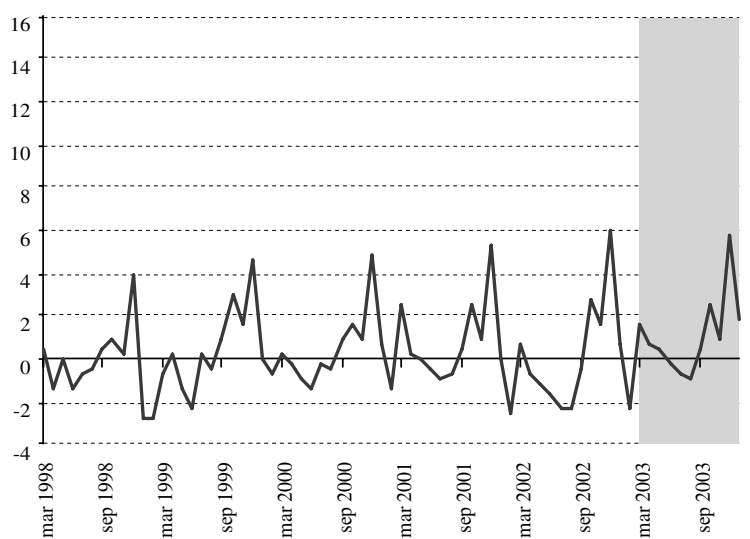

Fuente: Banco Central de Chile.

En el gráfico 3 se comparan series expresadas sobre la base del año 2003. Solo se analiza el período comprendido entre enero de 2003 y diciembre de 2006. Se escoge este período porque solo existen datos con base en 2003 desde enero de ese año y porque se supone que los últimos datos definitivos son los de diciembre de 2006.

En el gráfico 3 se observa un comportamiento de las revisiones distinto al capturado en las gráficos 1 y 2. En efecto, en el gráfico 3 las revisiones aparecen de menor magnitud y con un patrón estacional bastante menos acentuado que en el caso del gráfico 2.

Los resultados de los gráficos 1,2 y 3 son interesantes, puesto que configuran un panorama en el que las revisiones siguen procesos muy distintos. Estas han mostrado una tendencia a la disminución de su magnitud y sesgo en el tiempo, lo que es totalmente

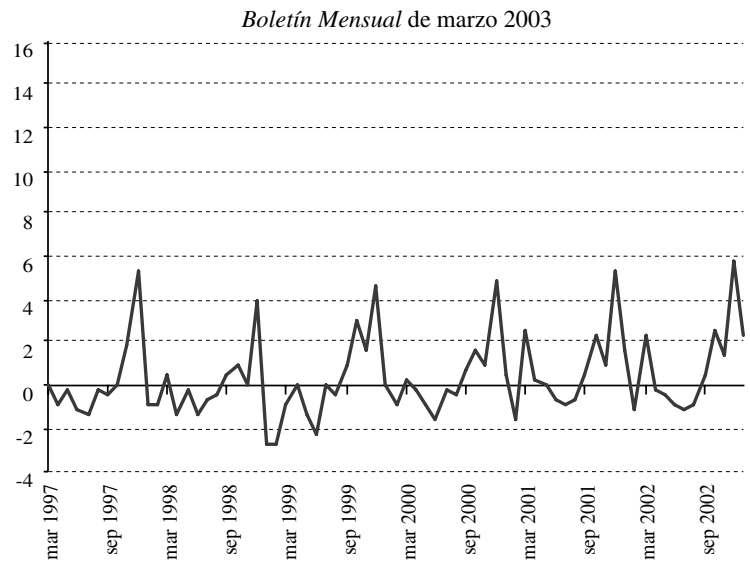

Boletín Mensual de marzo 2005

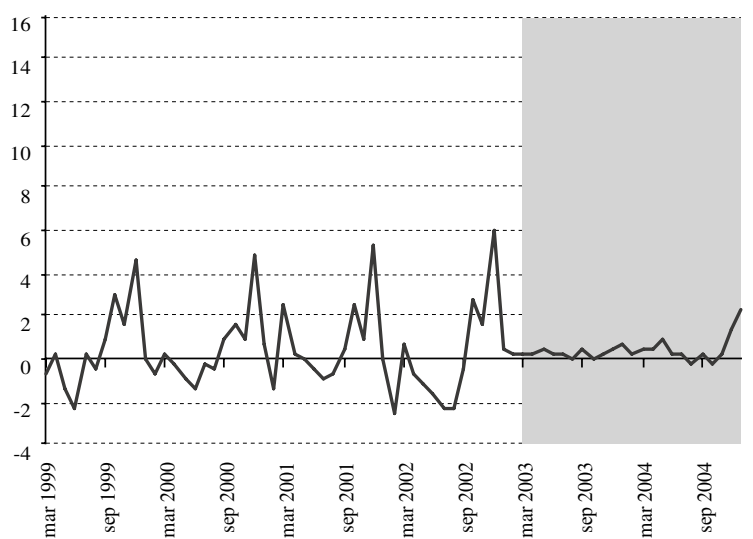

concordante con el resultado análogo mostrado por Pincheira (2010) en relación con el crecimiento del PIB anual. En la medida en que el proceso de revisiones se mantenga propenso a moderar su magnitud y sesgo, es indudable que la incertidumbre proveniente del hecho de no contar con datos definitivos en tiempo real tenderá a mitigarse, y tal vez a desaparecer. No obstante, el mismo análisis aquí realizado sugiere que esta fuente de incertidumbre ha sido importante en la muestra considerada en el presente trabajo ${ }^{8}$.

\footnotetext{
8 Para tener otra perspectiva respecto de la magnitud actual de las revisiones, también se calcularon las diferencias entre las series en tiempo real y las revisadas, expresadas en variaciones a 12 meses. En algunos meses las diferencias entre ambas series excedían los 200 puntos base, lo que indica que el tamaño de las revisiones es relevante.
} 
Evolución de la diferencia entre el IMACEC final y en tiempo real (IF - ITR) (Índice año base $2003=100$ )
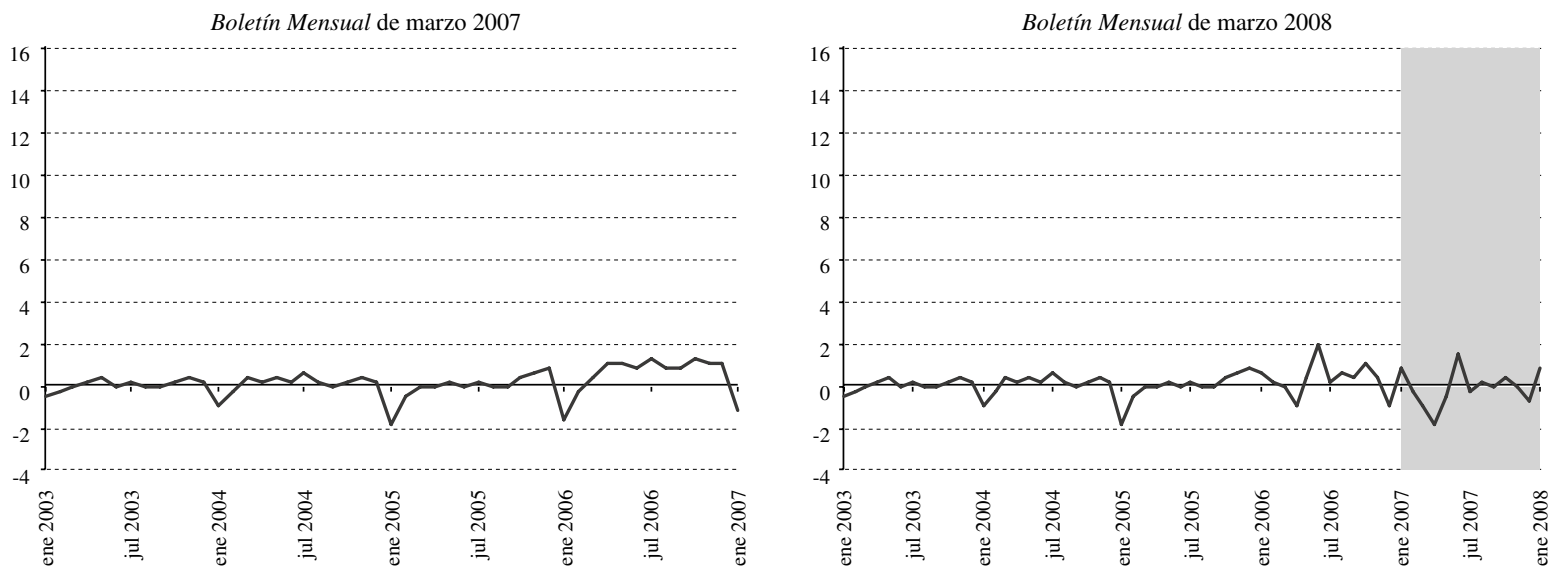

Boletín Mensual de marzo 2009

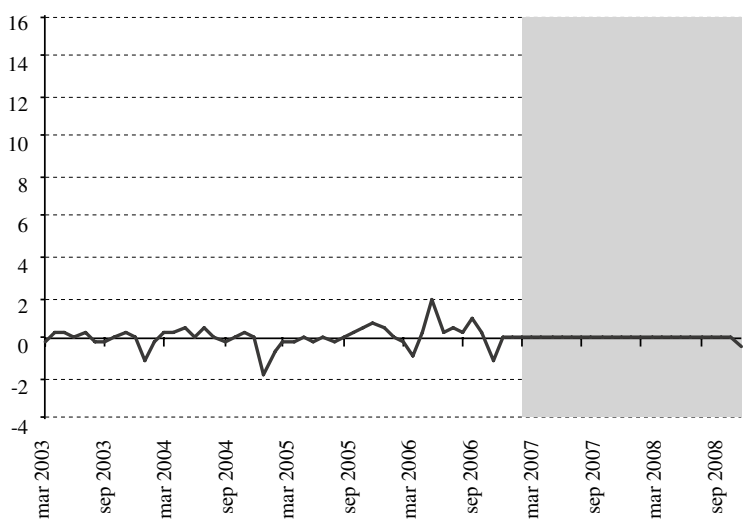

Fuente: Banco Central de Chile.

\section{Evaluación predictiva dentro de muestra: datos revisados}

El primer ejercicio aquí efectuado consiste en estimar las ecuaciones (1) a (4) en 152 ventanas rodantes de 71 observaciones cada una, de manera de tener una idea de la evolución del parámetro $\gamma$ correspondiente a cada medida de actividad considerada. La primera ventana capta los datos mensuales del IMACEC entre enero de 1991 y noviembre de 1996. Este primer ejercicio se realiza con datos revisados y disponibles en la página web del всCH a diciembre del 2009. Incluso así se ha procedido a desestacionalizar la serie del IMACEC y a calcular la brecha de producto mediante el filtro de Hodrick-Prescott en cada ventana de estimación, a fin de evitar incorporar información futura en las estimaciones.
Se ha considerado que el último dato que no sufrirá más revisiones es el correspondiente a diciembre de 2006. Por ello, los gráficos siguientes tienen un sombreado a partir de enero de 2007, indicando que los valores a partir de esos meses incluyen datos no definitivos. Cada modelo se estima con ocho variantes. En estas variantes se consideran distintos números de rezagos de la inflación (de 1 a 4 rezagos), más la inclusión o exclusión de la variable "meta de inflación". Una estimación robusta del parámetro $\gamma$ se obtiene al tomar el promedio bayesiano sobre las ocho variantes de cada modelo considerado. Con este propósito se utilizan las expresiones mostradas en el anexo C de Pincheira y Calani (2010), sobre la base de estimaciones HAC (consistentes con la heterocedasticidad y autocorrelación) de las varianzas de los parámetros individuales de cada modelo de acuerdo con el método 
de Newey y West (1987 y 1994). Asimismo, se calculan varianzas robustas a la incertidumbre con respecto al modelo de acuerdo con el promedio bayesiano, y de esta forma se construyen estadísticos de tipo $t$ asintóticamente normales. La evolución del parámetro $\gamma$ en los modelos 1 y 3 para horizontes de 1, 3, 6, 9 y 12 meses, así como la de sus valores $p$, se observa en los gráficos 4 y 5 .

La curva de mayor grosor representa la estimación robusta del parámetro $\gamma$ asociado a la variable de actividad que se está usando. La línea delgada indica el valor $p$ asociado al coeficiente. La línea recta entrecortada marca el nivel de significancia del 10\%. Esto quiere decir que el parámetro estimado será estadísticamente significativo, con un nivel de confianza del $90 \%$ o superior, cada vez que la línea delgada se encuentre por debajo de la línea recta entrecortada. Se omiten gráficas del parámetro $\gamma$ para los modelos 2 y 4 , porque son muy similares a los del gráfico 4 y no agregan información sustancialmente distinta a la ya mostrada.

Tal vez lo más interesante de todos los gráficos es que en ellos se aprecia una significancia estadística "episódica" del parámetro asociado a la variable de actividad. En otras palabras, la significancia estadística de este parámetro varía en el tiempo de manera que períodos de alta significancia son sucedidos por períodos de baja significancia. Por lo demás, esta alternancia suele darse en repetidas oportunidades durante el período muestral. La única excepción a esta frecuente alternancia se observa en el modelo 3, que denota una oscilación bastante más reducida en la significancia estadística. En el cuadro 1 se ilustra el carácter "episódico" del parámetro asociado a la variable de actividad, al señalar el porcentaje de las ventanas de estimación en las que este parámetro es significativo al $10 \%$. Se aprecia que este porcentaje varía según el modelo y el horizonte de predicción que se considere. En particular, se observa que la mayor frecuencia de significancia estadística se concentra en el horizonte de predicción de un mes para todos los modelos. Esta frecuencia oscila entre el $57,9 \%$ y el $84,2 \%$. Por el contrario, la menor frecuencia de significancia se concentra en los horizontes predictivos más largos, de 9 y 12 meses. En estos horizontes, la variable de actividad aparece estadísticamente significativa en menos de la mitad de las ventanas rodantes de estimación. Al comparar el comportamiento de los modelos, resalta que los resultados de las especificaciones 1 y 2 son muy similares. El modelo 3, en tanto, destaca por tener la frecuencia de significancia más baja en los dos primeros horizontes. A su vez, el modelo 4 sobresale por presentar la frecuencia de significancia más alta en proyecciones de un mes hacia adelante, y las frecuencias más bajas en horizontes de 6, 9 y 12 meses hacia adelante.

\begin{tabular}{|c|c|c|c|c|}
\hline \multirow{2}{*}{ CUADR } & \multicolumn{4}{|c|}{$\begin{array}{l}\text { Ventanas rodantes en que el parámetro } \\
\text { asociado a la actividad económica es } \\
\text { significativo al } 10 \% \text { a } \\
\text { (En porcentajes) }\end{array}$} \\
\hline & Modelo 1 & Modelo 2 & Modelo 3 & Modelo 4 \\
\hline$h=1$ & 73,0 & 71,1 & 57,9 & 84,2 \\
\hline$h=3$ & 50,0 & 52,6 & 43,4 & 44,1 \\
\hline$h=6$ & 46,1 & 46,7 & 41,4 & 17,1 \\
\hline$h=9$ & 36,2 & 34,2 & 33,6 & 16,4 \\
\hline$h=12$ & 44,1 & 42,8 & 35,5 & 15,1 \\
\hline
\end{tabular}

Fuente: elaboración propia.

a Datos definitivos: enero de 1991 a junio de 2009.

Finalmente, también es importante referirse a la magnitud de la estimación del parámetro $\gamma$. Se observa que, en general, la estimación de $\gamma$ es de un valor moderado o pequeño. Si bien su valor positivo más grande en todos los gráficos es de 1,34, cifra no despreciable, el promedio de las estimaciones obtenidas en todas las ventanas rodantes, para cada modelo y horizonte, no excede el valor de 0,23 . Estos números, más la inspección visual de los gráficos 4 y 5 , sugieren que el aporte predictivo de la variable de actividad en las ecuaciones (1) a (4) es moderado e inestable.

Todo esto configura un panorama en que el coeficiente asociado a la variable de actividad es, en general, "episódico" en términos de significancia estadística, y donde el estimador de este parámetro presenta inestabilidad y una magnitud moderada. Estos resultados son coherentes con la hipótesis atribuida a Michael McCracken, presentada en la Introducción, y también con aquellos resultados para los Estados Unidos de América en los que no se encontraba mayor predictibilidad con una serie de curvas de Phillips. En particular, este resultado es muy similar al que reportan Stock y Watson (2008), en el sentido de que la predictibilidad que entregan las versiones de curvas de Phillips hasta aquí analizadas también puede denominarse como "episódica". 
GRÁFICO 4

Evolución del parámetro y valor $p$ asociado a la actividad económica en la curva de Phillips del modelo 1, 1997-2009

(Datos definitivos)

Horizonte 1

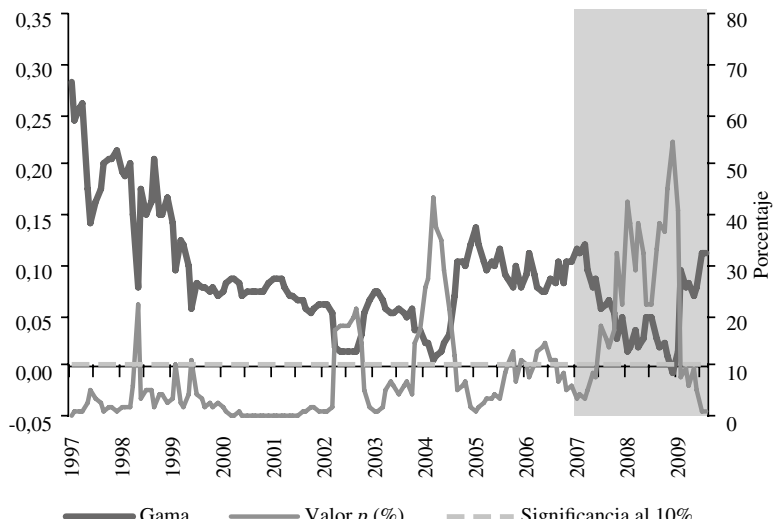

Horizonte 6

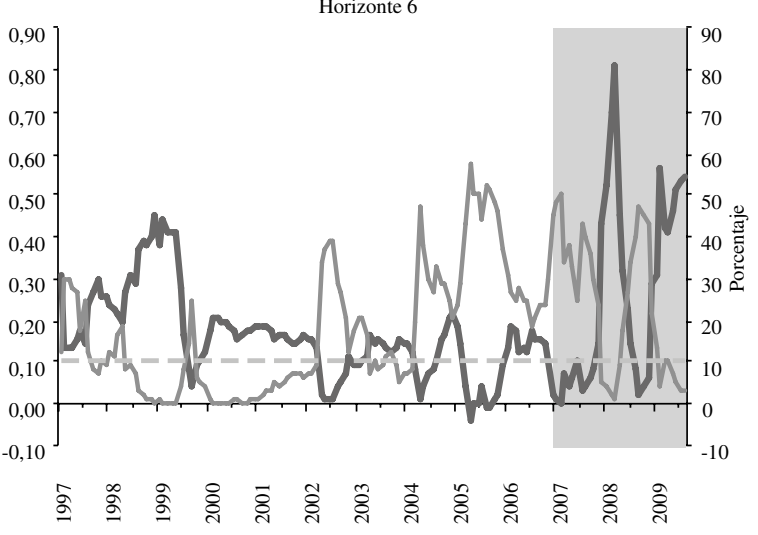

Horizonte 3

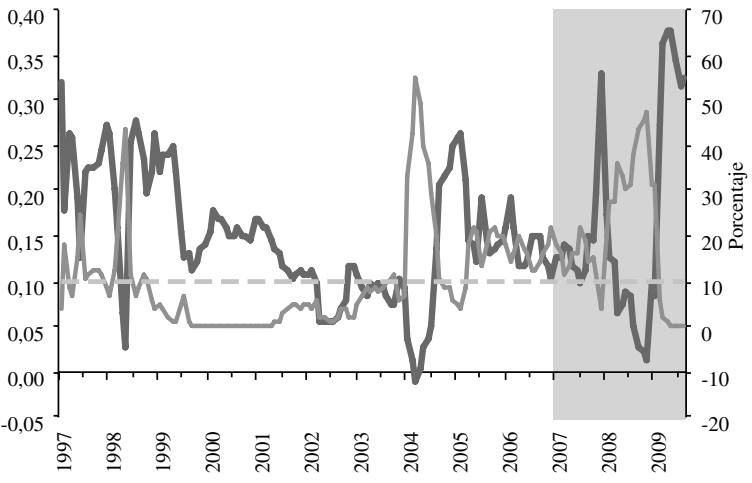

Gama $\longrightarrow$ Valor $p(\%) \quad-\quad-$ Significancia al $10 \%$

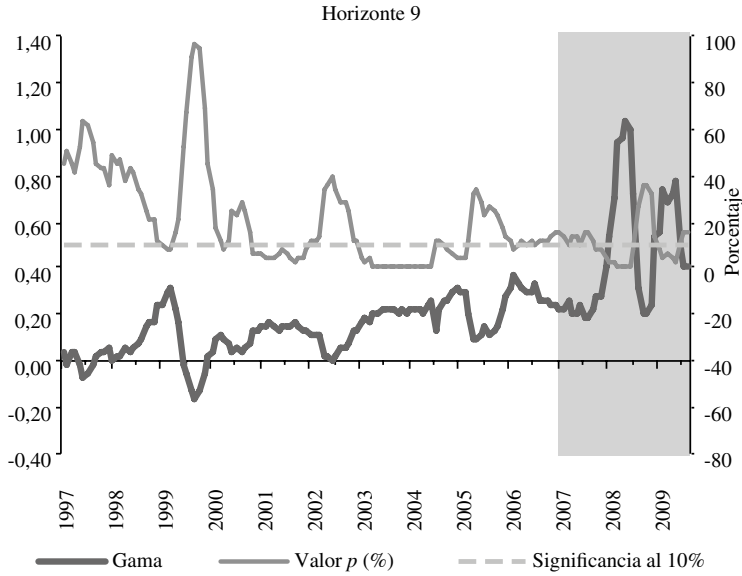

Horizonte 12

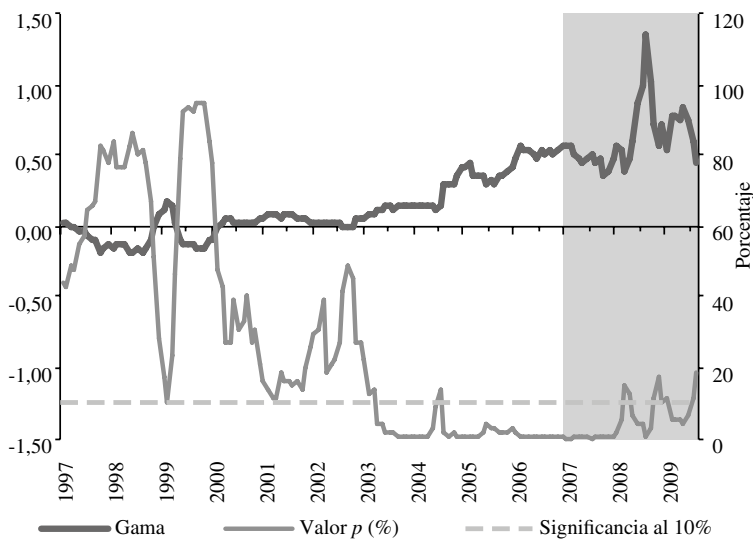

Fuente: elaboración propia. 
GRÁFICO 5

Evolución del parámetro y valor $p$ asociado a la actividad económica en la curva de Phillips del modelo 3, 1997-2009

(Datos definitivos)
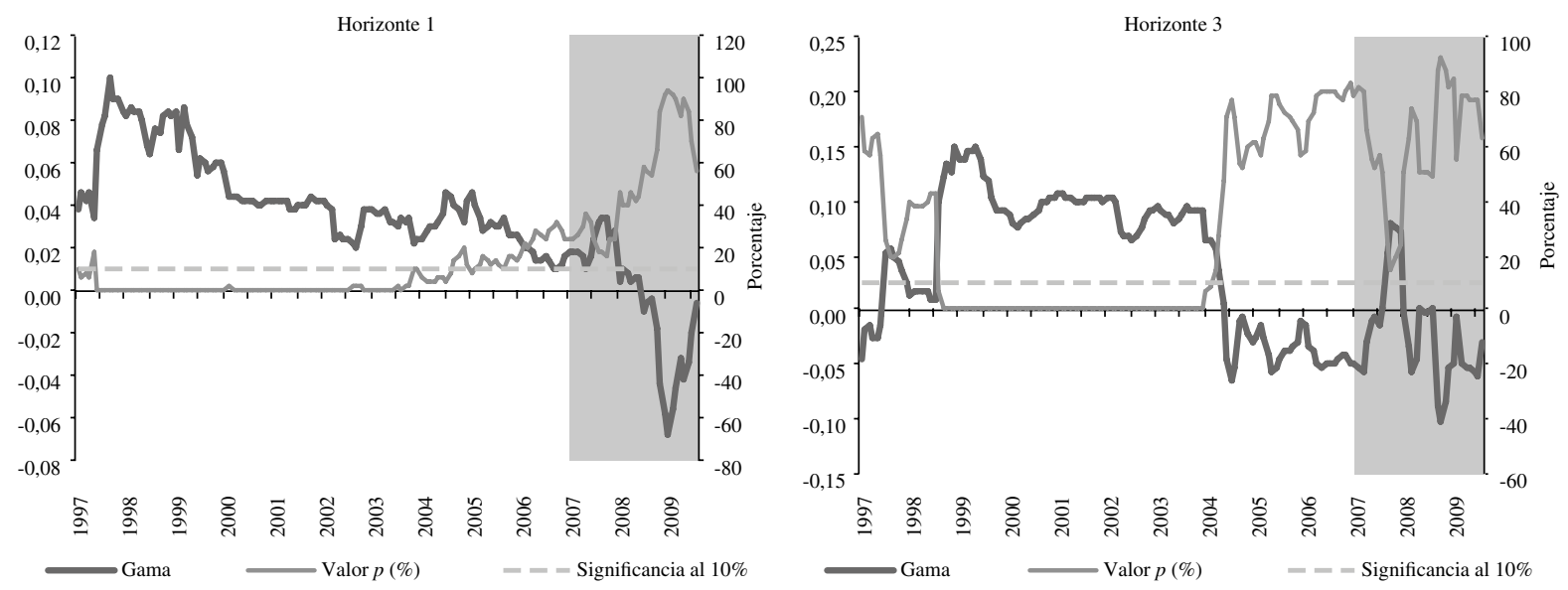

Horizonte 6
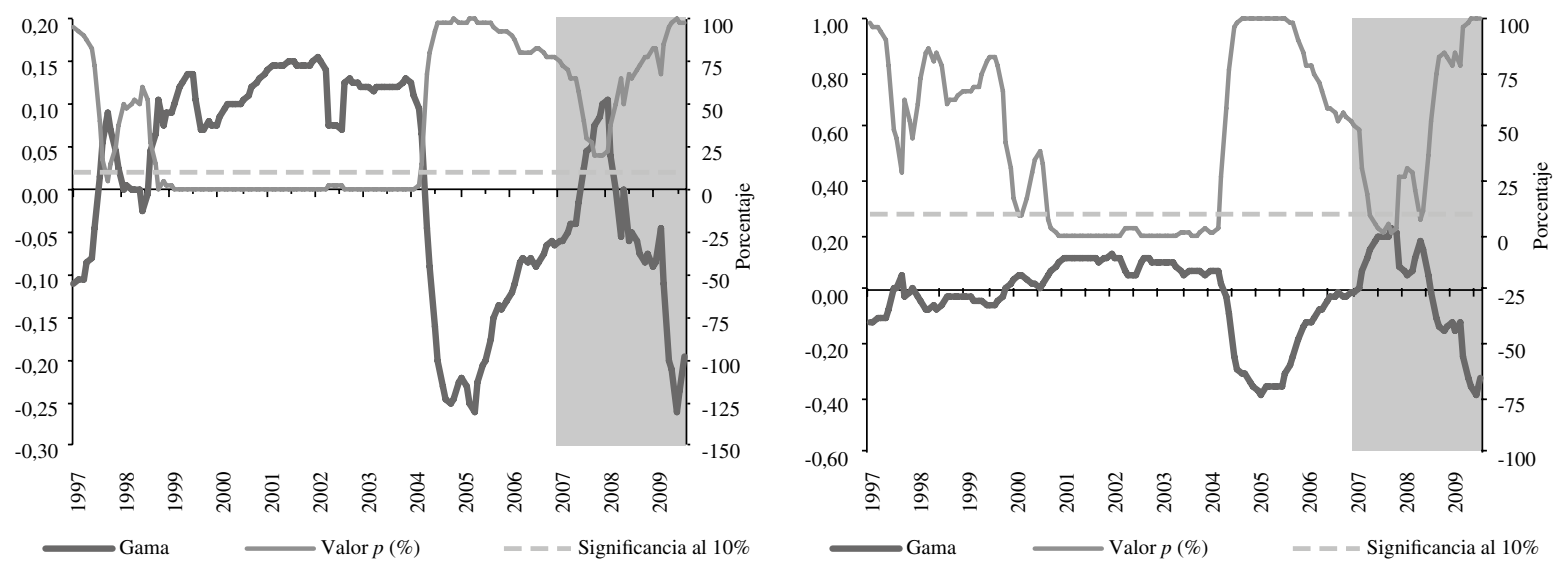

Horizonte 12

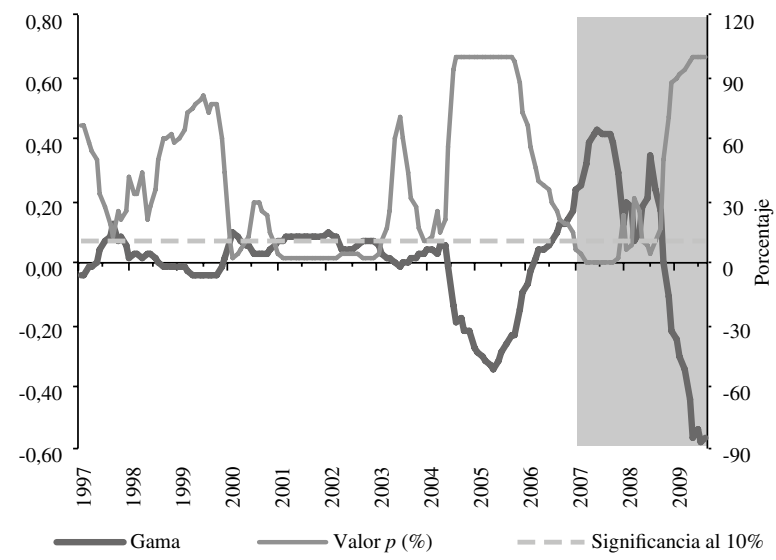

Fuente: elaboración propia. 


\section{Evaluación predictiva dentro de muestra: datos en tiempo real}

El análisis que se efectúa en esta subsección es análogo al de la subsección anterior, con la única y gran diferencia de que ahora las estimaciones y la construcción de la variable de actividad se realizan con datos en tiempo real. Esto se hace para evaluar si las variables de actividad en los modelos ( $1 \mathrm{al}$ 4) son útiles para generar buenos pronósticos de inflación que puedan ser utilizados por aquellos agentes encargados de tomar decisiones en tiempo real.

Al igual que en el análisis con datos revisados, también se aprecia en los gráficos 6 y 7 una significancia estadística "episódica" del parámetro asociado a la variable de actividad en los modelos 1 y 3 . Se omiten gráficos del parámetro $\gamma$ para los modelos 2 y 4 , porque son muy similares a los del modelo 1 y no agregan información sustancialmente distinta a la ya mostrada. El cuadro 2 es análogo al cuadro 1 en cuanto muestra el porcentaje de las ventanas de estimación en las que este parámetro es significativo al $10 \%$.

CUADRO 2

Ventanas rodantes en que el parámetro
asociado a la actividad económica es
significativo al $10 \%$
(Datos en tiempo real)

\begin{tabular}{lcccc}
\hline & Modelos 1 & Modelos 2 & Modelos 3 & Modelos 4 \\
\hline $\mathrm{h}=1$ & 65,8 & 65,1 & 73,0 & 73,0 \\
$\mathrm{~h}=3$ & 65,8 & 63,8 & 44,7 & 56,6 \\
$\mathrm{~h}=6$ & 63,2 & 60,5 & 39,5 & 39,5 \\
$\mathrm{~h}=9$ & 53,9 & 55,3 & 38,2 & 35,5 \\
$\mathrm{~h}=12$ & 48,7 & 50,0 & 40,8 & 28,9 \\
\hline
\end{tabular}

Fuente: elaboración propia.

Resalta que este porcentaje varía según el modelo y el horizonte de predicción que se considere en forma similar a lo que ocurría en el caso en que se utilizan datos definitivos. En particular, se observa que nuevamente la mayor frecuencia de significancia estadística se concentra en el horizonte de predicción de un mes para todos los modelos. Esta frecuencia oscila entre el $65,1 \%$ y el $73 \%$. Por otra parte, la menor frecuencia de significancia se concentra nuevamente en los horizontes predictivos más largos de 9 y 12 meses. En estos horizontes, la variable de actividad aparece estadísticamente significativa en a lo más el 55,3\% de las ventanas rodantes de estimación. Al comparar el comportamiento de los modelos, también se comprueba que los resultados de las especificaciones (1) y (2) son muy similares. El modelo 3, en tanto, ahora ya no destaca por tener la frecuencia de significancia más baja en los dos primeros horizontes; de hecho, comparte el primer lugar con el modelo 4 en cuanto a frecuencia de significancia estadística en el horizonte de un mes hacia adelante. El modelo 4 también destaca por presentar la frecuencia de significancia más baja en horizontes de 9 y 12 meses.

Con respecto a la magnitud de la estimación del parámetro $\gamma$, los resultados son también similares a los obtenidos con datos definitivos. De hecho, los gráficos 6 y 7 dan cuenta de un valor de estimación de $\gamma$ pequeño o moderado, alcanzando un máximo puntual de 1,25, pero promediando a través de las estimaciones obtenidas en todas las ventanas rodantes un valor que no excede de 0,30. Estos números, más la inspección visual de los gráficos 6 y 7, sugieren que el aporte predictivo de la variable de actividad en las ecuaciones (1) a (4) es moderado e inestable cuando dicha variable es introducida con datos en tiempo real, en un resultado muy similar al obtenido con datos finales.

Hasta ahora se ha hecho una comparación de carácter general o global entre los resultados asociados al parámetro de actividad en las ecuaciones (1) a (4), cuando esta estimación se realiza con datos definitivos y en tiempo real. Se han visto varias similitudes de carácter general entre estas dos estimaciones. Sin embargo, esto no debe confundirse con una afirmación de irrelevancia con respecto a la naturaleza de los datos con que se estiman las especificaciones (1) a (4). De hecho, tanto las estimaciones de $\gamma$ como los pronósticos de inflación derivados de una misma ecuación, estimada en un mismo período muestral pero con datos en tiempo real o definitivos, pueden diferir sustancialmente. Esto se observa en los gráficos 8 y 9 , que permiten ver que por ciertos períodos la estimación del parámetro $\gamma$ y las proyecciones de inflación a 12 meses que se derivan de las ecuaciones (1) a (4) parecen ser muy distintas cuando la estimación se hace con datos en tiempo real o definitivos. En efecto, las diferencias en los pronósticos de inflación ocasionalmente han llegado a superar los 100 puntos base y se observan con cierta frecuencia diferencias del orden de los 50 puntos base, que si bien no son enormes, tampoco parecen ser despreciables.

En síntesis, este análisis sugiere que, en promedio, el aporte marginal de la variable de actividad en el pronóstico de inflación es episódico, de magnitud moderada e inestable en el tiempo. Esta conclusión es robusta a la naturaleza de los datos con los que las curvas de Phillips son estimadas en este trabajo. No obstante, los pronósticos puntuales de inflación, asî como cada estimación del parámetro que acompaña a la variable de actividad, pueden cambiar significativamente dependiendo de si la ecuación en cuestión es estimada con datos revisados o en tiempo real. 
GRÁFICO 6

Evolución en tiempo real del parámetro y valor $p$ asociado a la actividad económica en la curva de Phillips del "modelo 1"a (En porcentajes)
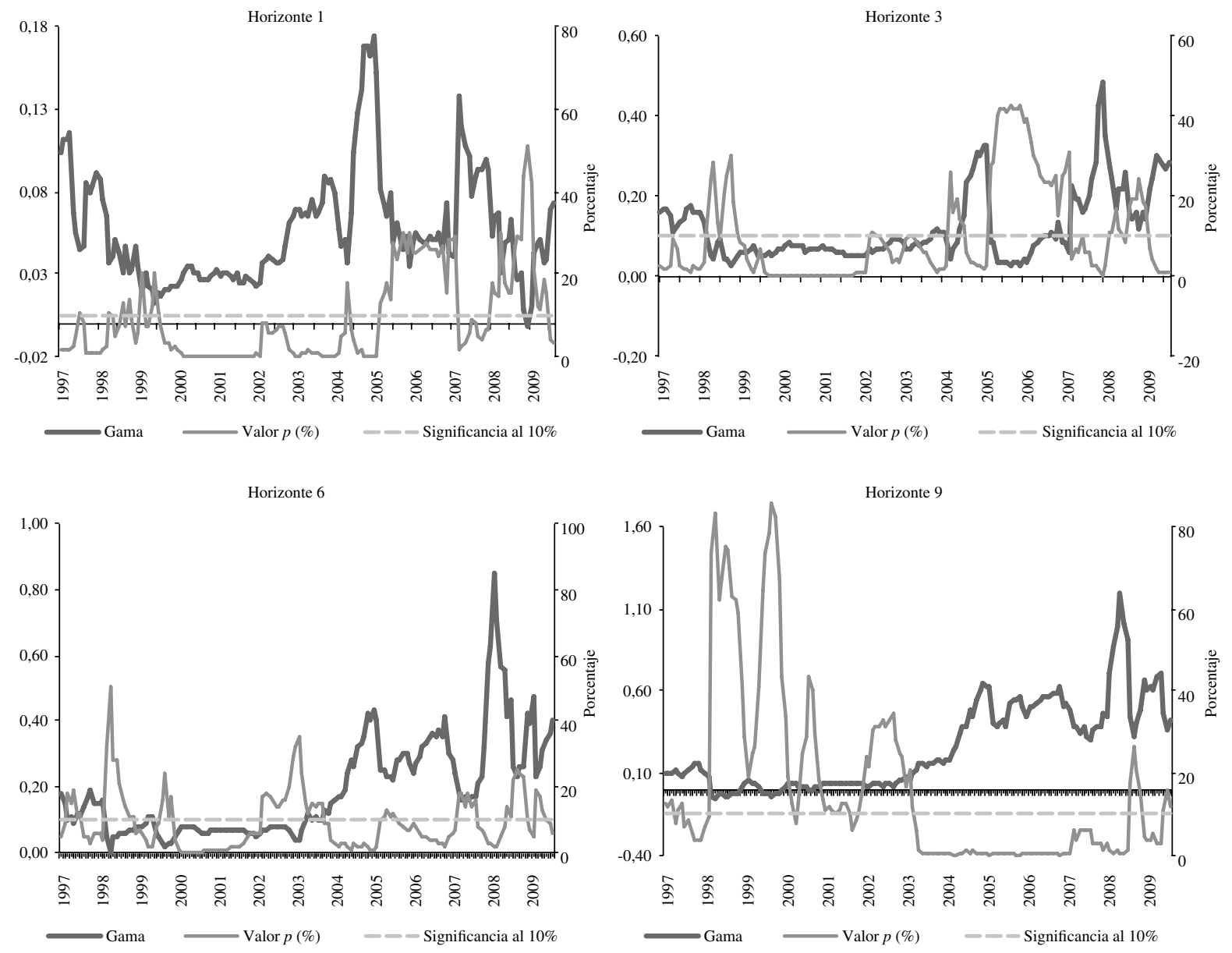

Horizonte 12

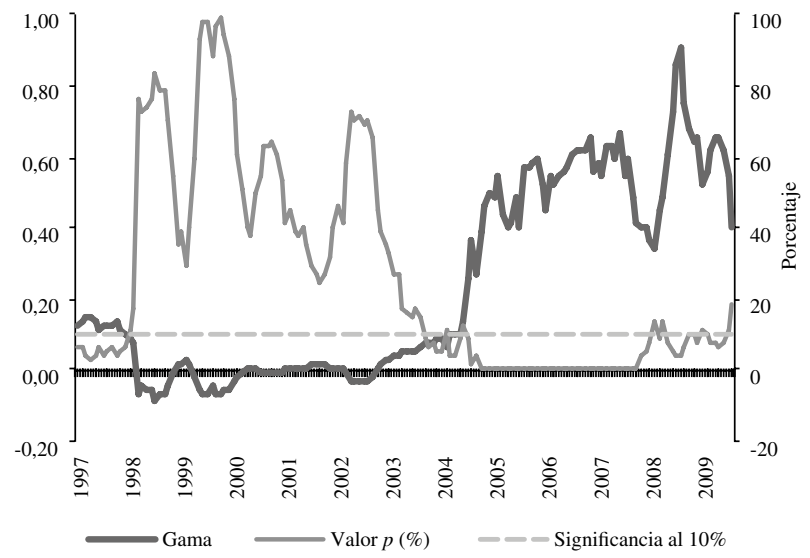

Fuente: elaboración propia.

a Datos desde enero de 1991 a junio de 2009. 
GRÁFICO 7

Evolución en tiempo real del parámetro y valor $p$ asociado a la actividad económica en la curva de Phillips del "modelo 3"

(Datos en tiempo real)
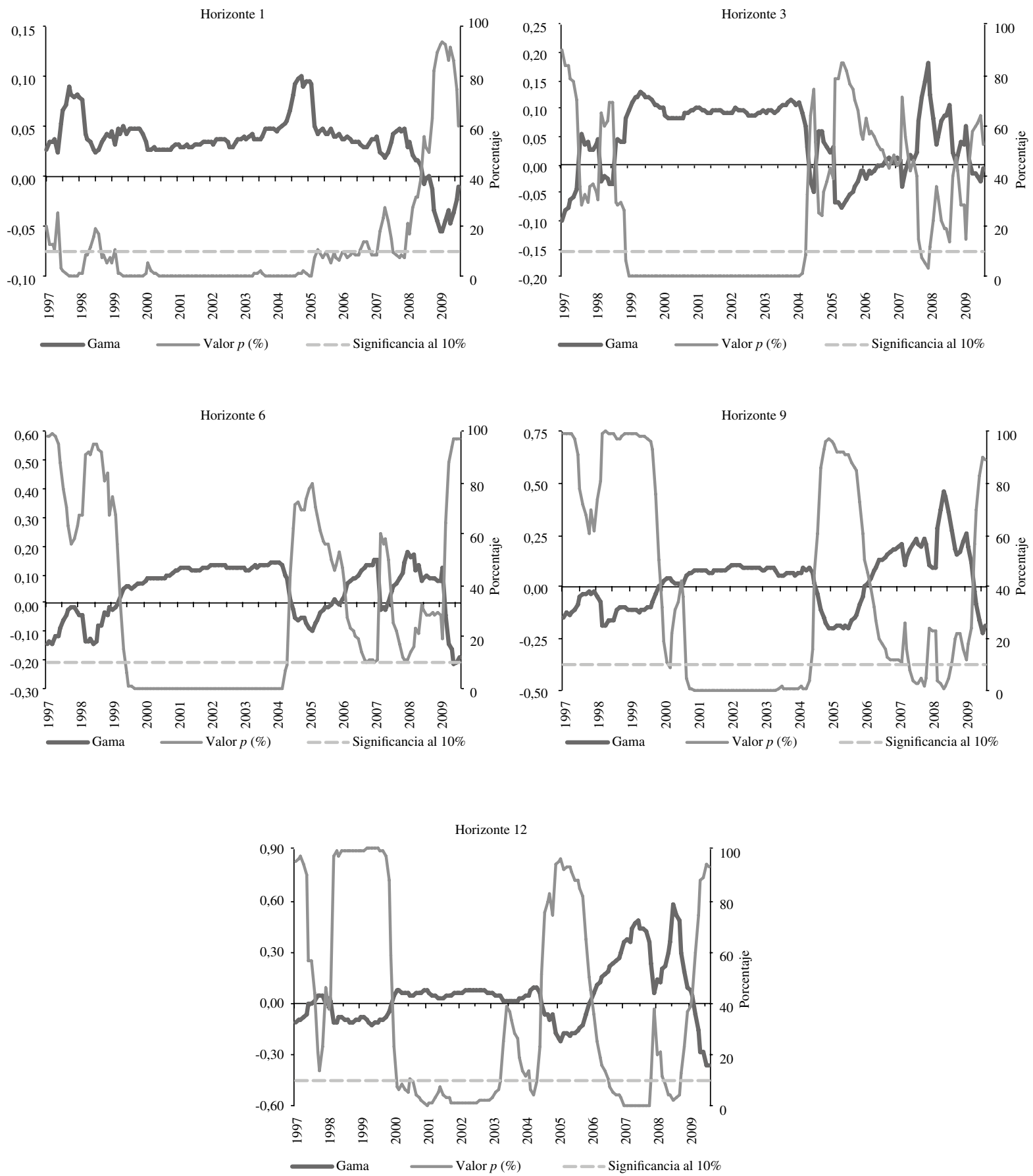

Fuente: elaboración propia. 
GRÁFICO 8

Diferencia entre las estimaciones de $\gamma$ en una misma ecuación estimada con datos definitivos y en tiempo real

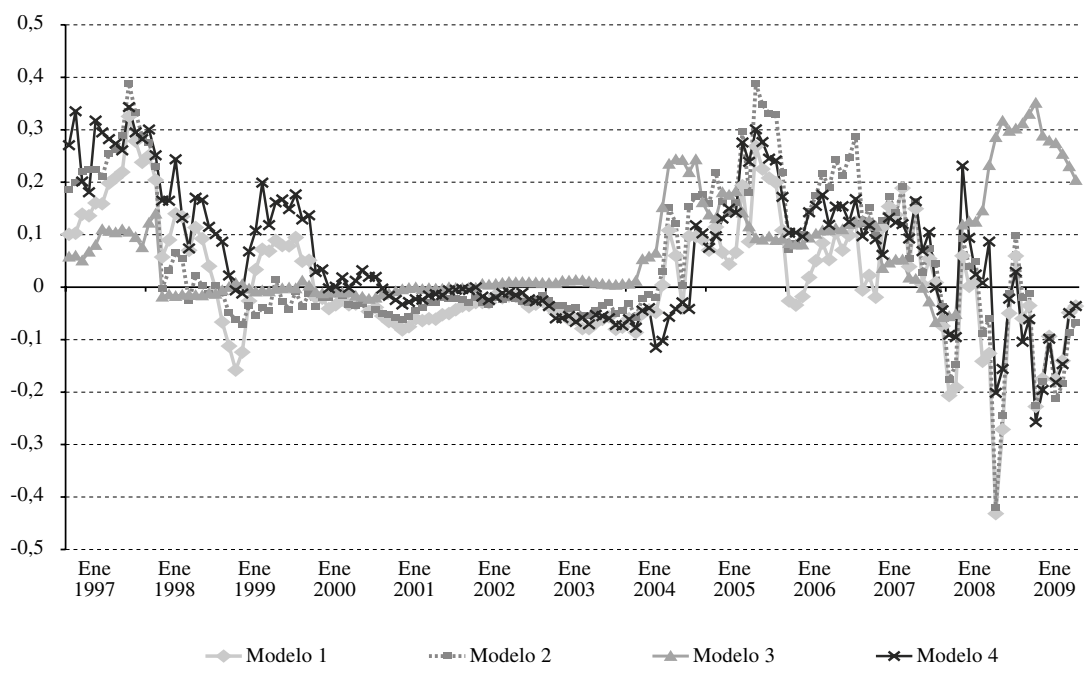

Fuente: elaboración propia.

GRÁFICO 9

Diferencia entre los pronósticos de inflación a 12 meses de una misma ecuación estimada con datos definitivos y en tiempo real

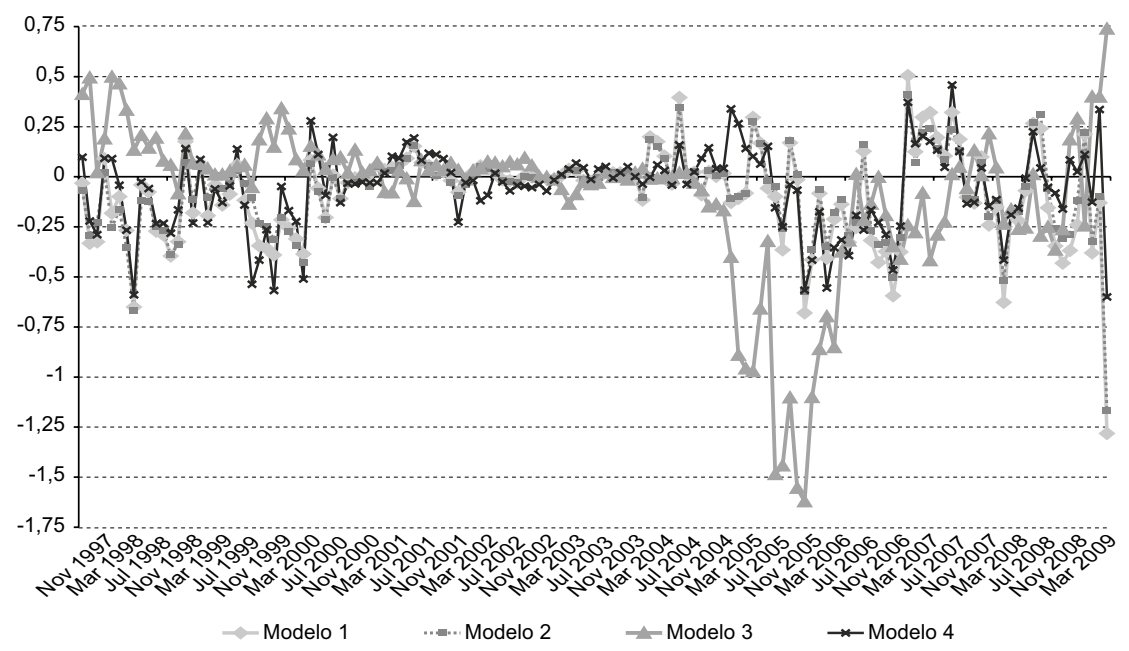

Fuente: elaboración propia. 


\section{Resultados complementarios fuera de muestra}

Los resultados presentados en las subsecciones anteriores corresponden a simples regresiones dentro de muestra. El carácter "episódico" e inestable del estimador del coeficiente asociado a la variable de actividad económica, así como su magnitud moderada, hacen presagiar que en ejercicios de predicción fuera de muestra, el aporte predictivo de las medidas de actividad económica debería ser mínimo. En el cuadro 3 se confirma este presagio. En este cuadro se evidencia el cociente de la raíz del error cuadrático medio de proyección fuera de muestra de cada uno de los modelos (1 al 4), estimados con y sin variable de actividad y en los cinco horizontes que se han considerado en este trabajo: 1, 3, 6, 9 y 12 meses hacia adelante. El ejercicio predictivo se basa en la utilización de las mismas ventanas rodantes de 71 observaciones que fueron usadas para el análisis dentro de muestra. Cabe señalar que para esta etapa se consideraron especificaciones con cuatro rezagos para inflación. En general, se observa que la mayoría de las cifras en el cuadro 3 son menores que 1, indicando que la inclusión de la variable de actividad, en la mayoría de los casos, perjudica la precisión predictiva de los modelos. Esto es coherente con la inestabilidad detectada en los parámetros asociados a la variable de actividad, a su carácter "episódico" y a su tamaño moderado.

En el cuadro 4 se complementa este análisis, comparando la raíz del error cuadrático medio de las curvas de Phillips con un modelo prototípico propuesto por Stock y Watson (2008) (para más detalles, véase el anexo) y algunos simples modelos de series de tiempo 9 . Se puede apreciar que los pronósticos provenientes de las curvas de Phillips son menos precisos que los mejores modelos de series de tiempo considerados en todos los horizontes. También es interesante destacar que la diferencia en precisión predictiva entre los modelos estimados con datos revisados y en tiempo real es muy pequeña, lo que es coherente con el mínimo aporte que en general poseen las variables de actividad aquí consideradas, cuya inclusión muchas veces es incluso perjudicial.

\footnotetext{
${ }^{9}$ Los modelos de serie de tiempo considerados son un camino aleatorio con constante y dos modelos SARIMA similares al modelo de aerolíneas (airline model) de Box y Jenkins (1970). Estos modelos SARIMA son descritos con gran detalle en Pincheira y García (2009), así como en Pincheira y Medel (2015), trabajos en los que también se muestra su excelente capacidad predictiva para la inflación de Chile y de un selecto grupo de países. Un breve resumen con las especificaciones SARIMA usadas en este documento se encuentra en los anexos.
}

\begin{tabular}{|c|c|c|c|c|c|c|c|}
\hline & & & \multicolumn{5}{|c|}{ Horizontes } \\
\hline & & & $\mathrm{h}=1$ & $h=3$ & $h=6$ & $h=9$ & $\mathrm{~h}=12$ \\
\hline Modelo 1 & $\begin{array}{l}\text { Tiempo real } \\
\text { Corregido }\end{array}$ & $\begin{array}{l}\text { sin meta } \\
\text { con meta } \\
\text { sin meta } \\
\text { con meta }\end{array}$ & $\begin{array}{l}0,98 \\
0,98 \\
0,97 \\
0,97\end{array}$ & $\begin{array}{l}0,94 \\
0,94 \\
0,95 \\
0,95\end{array}$ & $\begin{array}{l}0,93 \\
0,92 \\
0,94 \\
0,94\end{array}$ & $\begin{array}{l}0,90 \\
0,89 \\
0,90 \\
0,90\end{array}$ & $\begin{array}{l}0,97 \\
0,96 \\
0,96 \\
0,94\end{array}$ \\
\hline Modelo 2 & $\begin{array}{l}\text { Tiempo real } \\
\text { Corregido }\end{array}$ & $\begin{array}{l}\text { sin meta } \\
\text { con meta } \\
\text { sin meta } \\
\text { con meta }\end{array}$ & $\begin{array}{l}0,98 \\
0,98 \\
0,98 \\
0,98\end{array}$ & $\begin{array}{l}0,95 \\
0,94 \\
0,96 \\
0,96\end{array}$ & $\begin{array}{l}0,93 \\
0,93 \\
0,95 \\
0,94\end{array}$ & $\begin{array}{l}0,91 \\
0,90 \\
0,91 \\
0,90\end{array}$ & $\begin{array}{l}0,97 \\
0,96 \\
0,96 \\
0,95\end{array}$ \\
\hline Modelo 3 & $\begin{array}{l}\text { Tiempo real } \\
\text { Corregido }\end{array}$ & $\begin{array}{l}\text { sin meta } \\
\text { con meta } \\
\text { sin meta } \\
\text { con meta }\end{array}$ & $\begin{array}{l}1,00 \\
1,00 \\
0,98 \\
0,98\end{array}$ & $\begin{array}{l}0,99 \\
0,99 \\
0,99 \\
1,00\end{array}$ & $\begin{array}{l}0,99 \\
0,99 \\
1,00 \\
1,01\end{array}$ & $\begin{array}{l}0,97 \\
0,96 \\
0,97 \\
0,96\end{array}$ & $\begin{array}{l}1,02 \\
1,01 \\
0,97 \\
0,96\end{array}$ \\
\hline Modelo 4 & $\begin{array}{l}\text { Tiempo real } \\
\text { Corregido }\end{array}$ & $\begin{array}{l}\text { sin meta } \\
\text { con meta } \\
\text { sin meta } \\
\text { con meta }\end{array}$ & $\begin{array}{l}0,99 \\
0,99 \\
0,99 \\
1,00\end{array}$ & $\begin{array}{l}0,97 \\
0,97 \\
0,97 \\
0,97\end{array}$ & $\begin{array}{l}0,96 \\
0,96 \\
0,97 \\
0,97\end{array}$ & $\begin{array}{l}0,95 \\
0,95 \\
0,96 \\
0,95\end{array}$ & $\begin{array}{l}1,01 \\
1,00 \\
1,01 \\
0,99\end{array}$ \\
\hline
\end{tabular}

Fuente: elaboración propia.

a Ejercicio fuera de muestra entre noviembre de 1997 y junio de 2009. 


\begin{tabular}{|c|c|c|c|c|c|}
\hline & \multicolumn{5}{|c|}{ Horizontes } \\
\hline & $\mathrm{h}=1$ & $\mathrm{~h}=3$ & $\mathrm{~h}=6$ & $\mathrm{~h}=9$ & $\mathrm{~h}=12$ \\
\hline Camino aleatorio con constante & 0,48 & 1,04 & 1,75 & 2,20 & 2,53 \\
\hline SARIMA con constante & 0,35 & 0,90 & 1,50 & 1,81 & 2,00 \\
\hline SARIMA con constante y término autorregresivo & 0,34 & 0,90 & 1,51 & 1,82 & 2,01 \\
\hline Stock-Watson con constante & 0,39 & 1,04 & 1,79 & 2,26 & 2,55 \\
\hline Stock-Watson sin constante & 0,39 & 1,03 & 1,73 & 2,18 & 2,45 \\
\hline Phillips 1 con actividad definitiva & 0,44 & 1,00 & 1,79 & 2,39 & 2,48 \\
\hline Phillips 1 con actividad en tiempo real & 0,44 & 1,01 & 1,81 & 2,40 & 2,43 \\
\hline Phillips 2 con actividad definitiva & 0,44 & 0,99 & 1,78 & 2,37 & 2,47 \\
\hline Phillips 2 con actividad en tiempo real & 0,44 & 1,00 & 1,81 & 2,39 & 2,44 \\
\hline Phillips 3 con actividad definitiva & 0,45 & 1,00 & 1,72 & 2,24 & 2,49 \\
\hline Phillips 3 con actividad en tiempo real & 0,44 & 1,01 & 1,75 & 2,24 & 2,38 \\
\hline Phillips 4 con actividad definitiva & 0,47 & 0,99 & 1,78 & 2,17 & 2,25 \\
\hline Phillips 4 con actividad en tiempo real & 0,47 & 0,99 & 1,79 & 2,17 & 2,23 \\
\hline
\end{tabular}

Fuente: elaboración propia.

a Ejercicio fuera de muestra entre noviembre de 1997 y junio de 2009.

A los autores del presente artículo les parece interesante destacar dos observaciones adicionales en relación con ejercicios de inferencia estadística. Primero, cabe pensar que la aplicación de pruebas de habilidad predictiva del tipo Diebold y Mariano (1995); West (1996), y Giacomini y White (2006) no constituye un gran aporte para el propósito de este trabajo porque, básicamente, se ha visto que los modelos (1 al 4) entregan errores cuadráticos medios en general menores cuando son estimados sin variable de actividad, lo que garantiza que estas pruebas no pueden rechazar la hipótesis nula de igualdad de habilidad predictiva en favor de los modelos que incluyen variables de actividad. Es decir, en el peor caso, la hipótesis nula no se puede rechazar. Si bien es cierto que hay unos pocos casos en que las variables de actividad reducen la raíz del error cuadrático medio, la disminución nunca es superior al $2 \%$. Incluso si reducciones de este tamaño tuvieran significancia estadística, sería difícil sostener su significancia económica, lo que lleva a desistir de implementar ejercicios de inferencia que $a$ priori no se cree que puedan aportar significativamente a las conclusiones de este trabajo.

Segundo, y tal como se discute en Clark y West (2006 y 2007) y Pincheira (2013), esta comparación de errores cuadráticos medios no necesariamente implicaría que las variables de actividad no tuvieran un aporte que hacer a la predicción de inflación. Esto se debe a que la comparación de errores cuadráticos medios entre modelos anidados, en general, favorece al modelo con menos parámetros por estimar. No obstante, en este trabajo no solo se ha hecho un cálculo de error cuadrático medio, sino que también se ha visto en regresiones dentro de muestra el inestable y moderado aporte predictivo de las variables de actividad. En síntesis, ambos análisis, dentro y fuera de muestra, son coincidentes en señalar un bajo aporte de las variables de actividad para predecir inflación, al menos en el contexto de los modelos ( 1 al 4) aquí utilizados. 


\section{V}

\section{Un breve análisis de robustez}

\section{Modelos con cambio de régimen}

Ya se ha señalado anteriormente que el objetivo del presente estudio es evaluar si algunas medidas de actividad económica tienen capacidad para predecir inflación, en el contexto de simples versiones retrospectivas de curvas de Phillips del tipo backward-looking, lo que va en línea con una literatura internacional relativamente reciente ejemplificada en los trabajos de Stock y Watson (2008); Rossi y Sekhposyan (2010); Clark y McCracken (2006), y Ciccarelli y Mojon (2010).

No obstante lo anterior, es claro que existe una infinidad de especificaciones alternativas para predecir inflación, incluso dentro de la misma categoría de curvas de Phillips. Una línea de investigación paralela a la que aquí se ha seguido, se ha centrado en la utilización de modelos de cambio de régimen markovianos para caracterizar la inflación. Ejemplo de esto son los trabajos de Hostland (1995); Melo y Misas (1997); Amisano y Fagan (2013), y Pagliacci y Barráez (2010). Entre ellos, los más cercanos al presente artículo son los de Pagliacci y Barráez (2010) y Amisano y Fagan (2013).

Como un somero análisis de robustez, se realizan estimaciones dentro de muestra de curvas de Phillips backward-looking, como las especificadas en este trabajo, pero permitiendo la posibilidad de cambios de régimen endógenos en la línea del trabajo de Hamilton (1989) y de acuerdo con las siguientes especificaciones:

Modelo CR1:

$$
\begin{aligned}
\pi_{t+h} & =\delta_{1}^{s} \bar{\pi}_{t}+\alpha_{1}^{s}+\gamma_{1}^{s}\left(Y_{t-1}-Y_{t-1}^{*}\right) \\
& +\sum_{i=0}^{n} \varphi_{1, i}^{s} \pi_{t-i}+\varepsilon_{1, t+h}
\end{aligned} \quad, s=1,2
$$

Modelo CR2:

$$
\begin{gathered}
\pi_{t+h}=\delta_{2}^{s} \bar{\pi}_{t}+\alpha_{2}^{s}+\gamma_{2}^{s} 100\left(\ln \left[Y_{t-1}\right]-\ln \left[Y_{t-1}^{*}\right]\right) \\
+\sum_{i=0}^{n} \varphi_{2, i}^{s} \pi_{t-i}+\varepsilon_{2, t+h}
\end{gathered}
$$

Modelo CR3:

$$
\begin{gathered}
\pi_{t+h}=\delta_{3}^{s} \bar{\pi}_{t}+\alpha_{3}^{s}+\gamma_{3}^{s} 100\left(\ln \left[Y_{t-1}\right]-\ln \left[Y_{t-13}\right]\right), s=1,2 \\
+\sum_{i=0}^{n} \varphi_{3, i}^{s} \pi_{t-i}+\varepsilon_{3, t+h}
\end{gathered}
$$

Modelo CR4:

$$
\begin{aligned}
\pi_{t+h}-\pi_{t}= & \delta_{4}^{s} \bar{\pi}_{t}+\alpha_{4}^{s}+\gamma_{4}^{s} 100\left(\ln \left[Y_{t-1}\right]-\ln \left[Y_{t-1}^{*}\right]\right) \\
& +\sum_{i=0}^{n} \varphi_{4, i}^{s} \pi_{t-i}+\varepsilon_{4, t+h}
\end{aligned}, s=1,2
$$

Estas especificaciones alternativas son una generalización de las expresiones (1) a (4) originales, pero permitiendo dos regímenes para la inflación.

En el cuadro 5 se presentan los resultados de las estimaciones cuando se pronostica un mes hacia adelante. En ellas se ha impuesto exógenamente la posibilidad de que existan dos regímenes distintos que se diferencian por el subíndice $s$. Se observa que en todas las especificaciones, el coeficiente del término de actividad es pequeño. Además, se encuentra que solo en el modelo CR3 la variable de actividad posee un coeficiente estadísticamente significativo, lo que sucede en el régimen 2. En todos los otros casos, no hay significancia estadística. Es decir, en términos generales, se obtienen resultados similares a los que provienen de las especificaciones lineales: los términos de actividad solo tienen una capacidad predictiva episódica. Parece interesante destacar que el cuadro 5 también permite hacer una conjetura con respecto a las características que parecen diferenciar a un régimen del otro. Se constata que un régimen parece caracterizarse por una raíz unitaria, o al menos por un proceso con una raíz cercana a la unidad, mientras que el otro régimen pareciera tener una persistencia bastante menor. En todo caso, esto es solo una conjetura que sería interesante evaluar con más profundidad en trabajos futuros. Asimismo, los autores del presente estudio piensan que sería valioso investigar el comportamiento predictivo fuera de muestra de los modelos de cambio de régimen, lo que también se deja propuesto para una agenda de investigación futura. 
Parámetros y valores $p$ de curvas de Phillips $^{a}$ con cambio de régimen

\begin{tabular}{|c|c|c|c|c|c|c|c|c|}
\hline & \multicolumn{4}{|c|}{ Modelo CR1 } & \multicolumn{4}{|c|}{ Modelo CR2 } \\
\hline & \multicolumn{2}{|c|}{ Régimen 1} & \multicolumn{2}{|c|}{ Régimen 2} & \multicolumn{2}{|c|}{ Régimen 1} & \multicolumn{2}{|c|}{ Régimen 2} \\
\hline & Coeficiente & Valor $p$ & Coeficiente & Valor $p$ & Coeficiente & Valor $p$ & Coeficiente & Valor $p$ \\
\hline$\hat{\alpha}$ & 0,528 & 0,000 & $-0,378$ & 0,003 & $-0,237$ & 0,039 & 0,375 & 0,001 \\
\hline$\hat{\gamma}$ & 0,018 & 0,149 & $-0,011$ & 0,494 & $-0,017$ & 0,265 & 0,015 & 0,299 \\
\hline$\hat{\varphi}_{1}$ & 1,093 & 0,000 & 1,053 & 0,000 & 1,207 & 0,000 & 0,939 & 0,000 \\
\hline$\hat{\varphi}_{2}$ & 0,047 & 0,810 & $-0,159$ & 0,175 & $-0,149$ & 0,211 & $-0,071$ & 0,677 \\
\hline$\hat{\varphi}_{3}$ & $-0,132$ & 0,410 & 0,209 & 0,090 & 0,235 & 0,057 & $-0,059$ & 0,742 \\
\hline$\hat{\varphi}_{4}$ & $-0,048$ & 0,656 & $-0,230$ & 0,019 & $-0,287$ & 0,001 & 0,008 & 0,953 \\
\hline \multirow[t]{4}{*}{$\hat{\delta}$} & $-0,065$ & 0,022 & 0,183 & 0,000 & 0,052 & 0,033 & 0,073 & 0,053 \\
\hline & \multicolumn{4}{|c|}{ Modelo CR3 } & \multicolumn{4}{|c|}{ Modelo CR4 } \\
\hline & \multicolumn{2}{|c|}{ Régimen 1} & \multicolumn{2}{|c|}{ Régimen 2} & \multicolumn{2}{|c|}{ Régimen 1} & \multicolumn{2}{|c|}{ Régimen 2} \\
\hline & Coeficiente & Valor $p$ & Coeficiente & Valor $p$ & Coeficiente & Valor $p$ & Coeficiente & Valor $p$ \\
\hline$\hat{\alpha}$ & $-0,169$ & 0,176 & 0,184 & 0,278 & $-0,237$ & 0,039 & 0,375 & 0,001 \\
\hline$\hat{\gamma}$ & 0,017 & 0,370 & 0,053 & 0,092 & $-0,017$ & 0,266 & 0,015 & 0,303 \\
\hline$\hat{\varphi}_{1}$ & 1,151 & 0,000 & 0,884 & 0,000 & 0,207 & 0,016 & $-0,061$ & 0,644 \\
\hline$\hat{\varphi}_{2}$ & $-0,132$ & 0,273 & $-0,046$ & 0,782 & $-0,149$ & 0,212 & $-0,071$ & 0,679 \\
\hline$\hat{\varphi}_{3}$ & 0,209 & 0,075 & $-0,008$ & 0,948 & 0,235 & 0,058 & $-0,059$ & 0,761 \\
\hline$\hat{\varphi}_{4}$ & $-0,226$ & 0,007 & $-0,009$ & 0,918 & $-0,288$ & 0,001 & 0,008 & 0,959 \\
\hline$\hat{\delta}$ & 0,034 & 0,209 & 0,048 & 0,281 & 0,052 & 0,033 & 0,073 & 0,054 \\
\hline
\end{tabular}

Fuente: elaboración propia.

a Estimaciones backward-looking.

\section{Modelos con tipo de cambio}

Como un segundo análisis de robustez, se explora la posibilidad de que los resultados originales de este estudio se vean ensombrecidos debido a la omisión de variables relevantes. Si bien es cierto que el estimador de MCo es un estimador consistente al mejor predictor lineal, este parámetro poblacional, que hemos denotado $\beta^{*}$, puede diferir dependiendo del conjunto de información que se utilice para construir el pronóstico. En este sentido, se desea evaluar la robustez de los presentes resultados al incluir en las especificaciones originales una variable que es citada en la literatura como determinante de la inflación: el tipo de cambio (véase Pagliacci y Barráez, 2010; y en alguna medida también García-Solanes y Torrejón-Flores, 2012). Con esto, las especificaciones originales se modifican de la siguiente manera:
Modelo TC1:

$$
\begin{aligned}
& \pi_{t+h}=\delta_{1} \bar{\pi}_{t}+\alpha_{1}+\gamma_{1}\left(Y_{t-1}-Y_{t-1}^{*}\right) \\
& +\sum_{i=0}^{n} \varphi_{1, i} \pi_{t-i}+\theta_{1} L T C_{t}+\varepsilon_{1, t+h}
\end{aligned}
$$

Modelo TC2:

$$
\begin{aligned}
\pi_{t+h}= & \delta_{2} \bar{\pi}_{t}+\alpha_{2}+\gamma_{2} 100\left(\ln \left[Y_{t-1}\right]-\ln \left[Y_{t-1}^{*}\right]\right) \\
& +\sum_{i=0}^{n} \varphi_{2, i} \pi_{t-i}+\theta_{2} L T C_{t}+\varepsilon_{2, t+h}
\end{aligned}
$$

Modelo TC3:

$$
\begin{aligned}
\pi_{t+h}= & \delta_{3} \bar{\pi}_{t}+\alpha_{3}+\gamma_{3} 100\left(\ln \left[Y_{t-1}\right]-\ln \left[Y_{t-13}\right]\right) \\
& +\sum_{i=0}^{n} \varphi_{3, i} \pi_{t-i}+\theta_{3} L T C_{t}+\varepsilon_{3, t+h}
\end{aligned}
$$


Modelo TC4:

$$
\begin{gathered}
\pi_{t+h}-\pi_{t}=\delta_{4} \bar{\pi}_{t}+\alpha_{4}+\gamma_{4} 100\left(\ln \left[Y_{t-1}\right]-\ln \left[Y_{t-1}^{*}\right]\right) \\
+\sum_{i=0}^{n} \varphi_{4, i} \pi_{t-i}+\theta_{4} L T C_{t}+\varepsilon_{4, t+h}
\end{gathered}
$$

donde: $L T C_{t}=100\left(\ln \left[T C_{t}\right]-\ln \left[T C_{t-12}\right]\right)$

en tanto que $T C_{t}$ representa el tipo de cambio observado (pesos chilenos por dólar de los Estados Unidos de América) reportado por el $\mathrm{BCCH}$.

De esta manera, se desea analizar si el carácter episódico de los parámetros asociados a las variables de actividad se ve alterado o no al incluir la tasa de variación anual del tipo de cambio como predictor adicional. Con este fin se estiman las ecuaciones anteriores en las mismas ventanas rodantes reportadas en la sección IV.2. En el cuadro 6 se muestra el porcentaje de estas ventanas en las que el parámetro asociado a actividad resultó estadísticamente significativo con un nivel de confianza del 90\%. Es decir, el cuadro 6 es análogo al cuadro 1 presentado anteriormente.

Como puede observarse en el cuadro 6, el carácter episódico del parámetro asociado a la variable de actividad se mantiene. Se hace evidente que el porcentaje de ventanas en las que este parámetro es estadísticamente significativo oscila entre un $11,8 \%$ y el $64,5 \%$. El promedio general de los porcentajes en el cuadro 6 es de un $39,2 \%$, mientras que en el cuadro 1 es del 44,3\%. En síntesis, no se advierte que la incorporación del tipo de cambio como un predictor adicional altere cualitativamente los resultados. Incluso se podría llegar a argumentar que la inclusión del tipo de cambio deteriora la capacidad predictiva de la actividad, pero en general, al comparar los cuadros 1 y 6 se observa que los resultados son dependientes de la especificación. En efecto, se aprecia que en las ecuaciones (1), (2) y (4) la inclusión del tipo de cambio resta algo de significancia estadística al parámetro de la variable de actividad, mientras que en

\begin{tabular}{|c|c|c|c|c|}
\hline \multirow{2}{*}{ CUADRO } & \multicolumn{4}{|c|}{$\begin{array}{l}\text { Ventanas rodantes en que el parámetro } \\
\text { asociado a la actividad económica es } \\
\text { significativo al } 10 \%^{\mathrm{a}} \\
\text { (En porcentajes) }\end{array}$} \\
\hline & $\begin{array}{l}\text { Modelo } \\
\text { TC } 1\end{array}$ & $\begin{array}{l}\text { Modelo } \\
\text { TC } 2\end{array}$ & $\begin{array}{c}\text { Modelo } \\
\text { TC } 3\end{array}$ & $\begin{array}{c}\text { Modelo } \\
\text { TC } 4\end{array}$ \\
\hline$h=1$ & 50,0 & 50,0 & 64,5 & 57,9 \\
\hline$h=3$ & 46,7 & 46,1 & 41,4 & 28,3 \\
\hline$h=6$ & 40,1 & 40,8 & 56,6 & 11,8 \\
\hline$h=9$ & 23,0 & 23,7 & 55,9 & 17,8 \\
\hline $\mathrm{h}=12$ & 30,9 & 28,3 & 44,1 & 25,7 \\
\hline
\end{tabular}
la especificación (3) hay una tendencia opuesta.

Fuente: elaboración propia.

a Datos definitivos: enero de 1991 a junio de 2009. Especificaciones con tipo de cambio.

\section{VI}

\section{Resumen y conclusiones}

En este trabajo se han considerado cuatro familias de curvas de Phillips del tipo backward-looking para Chile en frecuencia mensual, y se ha evaluado la capacidad que ellas tienen para predecir inflación en horizontes de 1, 3, 6, 9 y 12 meses. En todas las especificaciones que se han considerado se incluye una variable de actividad que aparece rezagada respecto del último dato de inflación disponible, con el fin de emular la disponibilidad de información en tiempo real. Esto se hace así, porque los datos del IMACEC utilizados en este trabajo para construir nuestra variable de actividad son publicados con un mes de rezago con respecto al dato de inflación. El análisis de predictibilidad se hace tanto con cifras revisadas como con cifras disponibles en tiempo real, lo que permite evaluar la relación predictiva de tipo económico, así como la utilidad que tienen estas curvas de Phillips para generar proyecciones destinadas a los agentes vinculados a la toma de decisión.

Los resultados aquí obtenidos indican que la capacidad predictiva de estas curvas de Phillips es limitada, y más aún, que el aporte que hace la componente de actividad a esta predictibilidad es de tamaño moderado, muchas veces no estadísticamente significativo y bastante inestable. Esto es válido tanto para el análisis en tiempo real, como para el análisis con cifras revisadas.

Como un somero análisis de robustez, se ampliaron las especificaciones de modo de permitir la posibilidad de cambios de régimen de carácter markoviano, o la inclusión de la tasa de variación anual del tipo de cambio como predictor adicional. Estimaciones dentro de muestra de estas especificaciones ampliadas son consistentes con los resultados obtenidos en especificaciones lineales 
más simples, ya que la significancia estadística del parámetro asociado a la variable de actividad sigue siendo ocasional o episódica.

En parte de la literatura se señala que las evaluaciones de predictibilidad fuera de muestra adolecen de bajo poder con respecto a las evaluaciones dentro de muestra. Es por esa razón que en este estudio se han conducido ejercicios en ambos sentidos, obteniéndose resultados que apuntan en la misma dirección. De esta manera, parece más plausible atribuir la carencia de predictibilidad al inestable y moderado valor absoluto del coeficiente

ANEXO

\section{Metodología de Stock y Watson (2008)}

La curva de Phillips, por muchos años, ha sido una herramienta muy utilizada en la predicción de inflación. Sin embargo, los resultados empíricos que ofrece este documento, así como los de una importante rama de la literatura existente, no necesariamente son satisfactorios si se los compara con modelos en que solo se considera el pasado de la inflación. En este sentido, Stock y Watson (2008) presentan un modelo prototípico autorregresivo con la siguiente especificación:

$$
\pi_{t+h}^{(h)}-\pi_{t}^{(1)}=\alpha^{(h)} \sum_{i=0}^{n} \phi_{i}^{(h)} \Delta \pi_{t-i}^{(1)}+\varepsilon_{t+h}^{(h)}
$$

donde: $\pi_{t}^{(h)}=\frac{1200}{h}\left[\ln \left(P_{t}\right)-\ln \left(P_{t-h}\right)\right]$

corresponde a la inflación anualizada acumulada durante $h$ períodos a partir del instante $t ; \pi_{t}^{(1)}$ corresponde a la inflación anualizada acumulada durante solo 1 mes; la variable $\varepsilon_{t+h}^{(h)}$ representa perturbaciones no correlacionadas con la información disponible en $t$, y $\alpha^{(h)}$ representa una constante que puede variar dependiendo del período de acumulación de inflación utilizado.

El ejercicio aquí propuesto consiste en estimar este modelo prototípico, con constante y sin constante, en 152 ventanas rodantes de 71 observaciones cada una. La primera ventana toma datos del IPC desde enero de 1991 hasta noviembre de 1996. En cada ventana se estiman varios modelos, considerando distinto número de rezagos del diferencial de inflación $\Delta \pi_{t-i}^{(1)}$ (de 0 a 12 rezagos). Para determinar el mejor modelo dentro de cada ventana, se utilizó el criterio de Schwarz. Una vez seleccionado el modelo, con el número de rezagos de la variable independiente ya determinado, se realizan las proyecciones a distintos horizontes: 1, 3, 6, 9 y 12 meses asociado a las respectivas variables de actividad, que a problemas de poder de las pruebas estadísticas fuera de muestra.

Si bien los resultados de este trabajo muestran coherencia con los de varios artículos escritos para los Estados Unidos de América, son novedosos en cuanto evidencian una escasa capacidad predictiva de las curvas de Phillips tipo backward-looking en Chile. Será parte de una agenda futura determinar si estos interesantes resultados son heredados por otras versiones de curvas de Phillips.

hacia adelante. Como los resultados predictivos que entregan estos modelos corresponden a una función de la inflación, con simples pasos algebraicos se obtiene un pronóstico a distintos horizontes de la variable objetivo, que es la inflación acumulada en 12 meses. La raíz del error cuadrático fuera de muestra obtenida con esta metodología es presentada en el cuadro 4.

\section{Modelos SARIMA}

Aparte de los modelos de Stock y Watson detallados anteriormente, los modelos de serie de tiempo considerados en el cuadro 4 de este trabajo son un camino aleatorio con constante y dos modelos SARIMA similares al modelo de aerolíneas (airline model) de Box y Jenkins (1970). Estos modelos SARIMA son descritos con gran detalle en Pincheira y García (2009), así como en Pincheira y Medel (2015), artículos en los que también se muestra su excelente capacidad predictiva para la inflación de Chile y de un selecto grupo de países. En particular, los tres modelos aquí utilizados tienen las siguientes especificaciones:

Camino aleatorio con constante: $\pi_{t}=\alpha+\pi_{t-1}+\varepsilon_{t}$

SARIMA 1: $\pi_{t}=\alpha+\varepsilon_{t}-\theta \varepsilon_{t-1}-\vartheta \varepsilon_{t-12}+\vartheta \theta \varepsilon_{t-13}$

SARIMA 2: $\pi_{t}=\alpha+\rho \pi_{t-1}+\varepsilon_{t}-\theta \varepsilon_{t-1}$

$$
-\vartheta \varepsilon_{t-12}+\vartheta \theta \varepsilon_{t-13}
$$

donde: $\pi_{t}=100\left[\ln \left(I P C_{t}\right)-\ln \left(I P C_{t-12}\right)\right]$

El modelo denominado SARIMA 1 corresponde al modelo denominado "SARIMA con constante" en el cuadro 4. El modelo SARIMA 2 corresponde al modelo denominado "SARIMA con constante y término autorregresivo" en el mismo cuadro 4 . 


\section{Bibliografía}

Amisano, G. y G. Fagan (2013), "Money growth and inflation: A regime switching approach", Journal of International Money and Finance, vol. 33(C), Amsterdam, Elsevier.

Aguirre, A. y L.F. Céspedes (2004), "Uso de análisis factorial dinámico para proyecciones macroeconómicas", Economía Chilena, vol. $7, \mathrm{~N}^{\circ} 3$, Santiago de Chile, Banco Central de Chile.

Atkeson, A. y L. Ohanian (2001), "Are Phillips curves useful for forecasting inflation?", Quarterly Review, vol. 25, $\mathrm{N}^{\circ} 1$, Minneapolis, Banco de la Reserva Federal de Minneapolis.

Banco Central de Chile (2003), Modelos macroeconómicos y proyecciones del Banco Central de Chile 2003, Santiago de Chile.

Box, G. y G. Jenkins (1970), Time Series Analysis: Forecasting and Control, San Francisco, Holden-Day.

Box, G. y G. Tiao (1973), Bayesian Inference in Statistical Analysis, Nueva York, John Wiley and Sons.

Brock, W. y S. Durlauf (2001), "Growth empirics and reality", The World Bank Economic Review, vol. 15, N ${ }^{\circ}$ 2, Washington, D.C., Banco Mundial.

Céspedes, L.F., M. Ochoa y C. Soto (2005), "The new Keynesian Phillips curve in an emerging market economy: The case of Chile", Documento de Trabajo, $\mathrm{N}^{\circ} 355$, Santiago de Chile, Banco Central de Chile.

Chih-Chuan, Yeh (2009), "The Simultaneous Evolution of Growth and Inflation", documento presentado en la Conferencia Internacional de Economía Aplicada.

Chumacero, R. y F. Gallego (2002), "Trends and cycles in real-time", Estudios de Economía, vol. 29, $\mathrm{N}^{\circ}$ 2, Santiago de Chile, Universidad de Chile.

Ciccarelli, M. y B. Mojon (2010), "Global inflation", The Review of Economics and Statistics, vol. 92, $\mathrm{N}^{\circ} 3$, Cambridge, Massachusetts, The MIT Press.

Clark, T. y K. West (2007), "Approximately normal tests for equal predictive accuracy in nested models", Journal of Econometrics, vol. 138, $\mathrm{N}^{\circ} 1$, Amsterdam, Elsevier.

(2006), "Using out-of-sample mean squared prediction errors to test the martingale difference hypothesis", Journal of Econometrics, vol. 135, $\mathrm{N}^{\circ} 1-2$, Amsterdam, Elsevier.

Clark, T. y M. McCracken (2006), "The predictive content of the output-gap for inflation: Resolving in-sample and out-of-sample evidence", Journal of Money, Credit and Banking, vol. 38, $\mathrm{N}^{\circ}$ 5, Columbus, Ohio, The Ohio State University Press.

Diebold, F. y R. Mariano (1995), "Comparing predictive accuracy", Journal of Business and Economic Statistics, vol. 13, $\mathrm{N}^{\circ} 3$, American Statistical Association.

Estrella A. y J. Fuhrer (2003), "Monetary policy shifts and the stability of monetary policy models", Review of Economics and Statistics, vol. 85, $\mathrm{N}^{\circ} 1$, Cambridge, Massachusetts, The MIT Press.

Fisher I. (1973), "I discovered the Phillips curve", Journal of Political Economy, vol. 81, $\mathrm{N}^{\circ}$ 2, Chicago, University of Chicago Press.

(1926), "A statistical relationship between unemployment and price changes", International Labor Review, vol. $13, \mathrm{~N}^{\circ} 6$, Organización Internacional del Trabajo (OIT), junio.

Fuentes, R., F. Gredig y M. Larraín (2008), "La brecha de producto en Chile: Medición y evaluación”, Economía Chilena, vol. 11, $\mathrm{N}^{\circ}$ 2, Santiago de Chile, Banco Central de Chile.

García, M. y J.P. Medina (2008), "Efectos de primas financieras sobre la actividad agregada", Economía Chilena, vol. 12, $\mathrm{N}^{\circ} 3$, Santiago de Chile, Banco Central de Chile.

García-Solanes, J. y F. Torrejón-Flores (2012), "La fijación de metas de inflación da buenos resultados en América Latina", Revista de la CEPAL, $\mathrm{N}^{\circ} 106$ (LC/G.2518-P), Santiago de Chile.

Giacomini, R. y H. White (2006), "Tests of conditional predictive ability", Econometrica, vol. 74, $\mathrm{N}^{\circ}$ 6, Nueva York, The Econometric Society.
Hamilton, J. (1994), Time Series Analysis, Princeton, Princeton University Press.

(1989), "A new approach to the economic analysis of nonstationary time series and the business cycle", Econometrica, vol. 57, $\mathrm{N}^{\circ}$ 2, Nueva York, The Econometric Society.

Hansen, B. (2014), "Econometrics", Universidad de WisconsinMadison, inédito.

Hostland, D. (1995), "Changes in the inflation process in Canada: Evidence and implications", Working Paper, $N^{\circ} 1995-5$ Ottawa, Banco de Canadá.

Lansing, K. (2002), "Can the Phillips curve help forecast inflation", FRBSF Economic Letter, $\mathrm{N}^{\circ} 29$, San Francisco, Banco de la Reserva Federal de San Francisco.

Meese, R. y K. Rogoff (1983), "Empirical exchange rate models of the seventies. Do they fit out-of-sample?", Journal of International Economics, vol. 14, $\mathrm{N}^{\circ} 1-2$, Amsterdam, Elsevier.

Melo, L. y M. Misas (1997), "Análisis del comportamiento de la inflación trimestral en Colombia bajo cambios de régimen: Una evidencia a través del modelo 'Switching' de Hamilton", Borradores de Economía, ${ }^{\circ} 86$, Bogotá, Banco de la República de Colombia.

Morandé, F. y M. y Tejada (2008), "Fuentes de incertidumbre monetaria en la conducción de la política monetaria en Chile", Economía Chilena, vol. 11, $\mathrm{N}^{\circ}$ 3, Santiago de Chile, Banco Central de Chile.

Nadal de Simone, F. (2001), "Proyección de la inflación en Chile", Economía Chilena, vol. $4, \mathrm{~N}^{\circ} 3$, Santiago de Chile, Banco Central de Chile.

Newey, W. y K. West (1994), "Automatic lag selection in covariance matrix estimation", Review of Economic Studies, vol. $61, \mathrm{~N}^{\circ} 4$ Wiley Blackwell.

(1987), "A simple, positive, semi-definite, heteroskedasticity and autocorrelation consistent covariance matrix", Econometrica, vol. 55, $\mathrm{N}^{\circ} 3$, Nueva York, The Econometric Society.

Pagliacci, C. y D. Barráez (2010), “A Markov-switching model of inflation: looking at the future during uncertain times",

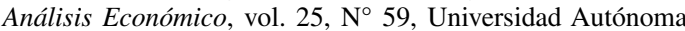
Metropolitana

Pedersen, M. (2013), "Extracting GDP signals from the monthly indicator of economic activity. Evidence from Chilean real-time data", Journal of Business Cycle Measurement and Analysis, vol. 2013(1), oECD Publishing.

Phillips, A. (1958), “The relation between unemployment and the rate of change in money wage rates in the United Kingdom, 1861-1957", Economica, vol. 25, Wiley, noviembre.

Pincheira P. (2013), "Shrinkage based tests of predictability", Journal of Forecasting, vol. 32, $\mathrm{N}^{\circ} 4$, Wiley.

(2010), "A real time evaluation of the Central Bank of Chile GDP growth forecasts", Money Affairs, vol. 23, $\mathrm{N}^{\circ} 1$, Centro de Estudios Monetarios Latinoamericanos.

Pincheira, P. y M. Calani (2010), "Communicational bias in monetary policy: Can words forecast deeds?", Economia, vol. $11, \mathrm{~N}^{\circ} 1$, Asociación de Economía de América Latina y el Caribe.

Pincheira, P. y A. García (2009), "En búsqueda de un buen benchmark predictivo para la inflación", Documento de Trabajo, $\mathrm{N}^{\circ} 514$, Santiago de Chile, Banco Central de Chile.

Pincheira, P. y C. Medel (2015), "Forecasting inflation with a simple and accurate benchmark: The case of the us and a set of inflation targeting countries", Czech Journal of Economics and Finance, vol. $65, \mathrm{~N}^{\circ} 1$

Rossi, B. y T. Sekhposyan (2010), "Have economic models' forecasting performance for us output changed over time, and when?", International Journal of Forecasting, vol. 26, $\mathrm{N}^{\circ} 4$ Amsterdam, Elsevier. 
Rudebusch, G. y L. Svensson (1999), "Policy rules for inflation targeting”, Monetary Policy Rules, J.B. Taylor (ed.), Chicago, University of Chicago Press.

Russell, B. y R. Chowdhury (2013), "Estimating United States Phillips Curves with expectations consistent with the statistical process of inflation", Journal of Macroeconomics, vol. 35, Amsterdam, Elsevier.

Samuelson, P. y R. Solow (1960), "Analytical aspects of anti-Inflation policy", American Economic Review, vol. 50, № 2, Nashville, Tennessee, American Economic Association.

Stock, J. y M. Watson (2008), "Phillips Curve inflation forecasts", NBER Working Paper, $\mathrm{N}^{\circ} 14322$, Cambridge, Massachusetts, National Bureau of Economic Research.

(1999), "Forecasting inflation", Journal of Monetary Economics, vol. 44, $\mathrm{N}^{\circ} 2$, Amsterdam, Elsevier.
(1998), "Diffusion indexes", NBER Working Paper, N ${ }^{\circ} 6702$, Cambridge, Massachusetts, National Bureau of Economic Research.

Taylor, J.B. (1993), "Discretion versus policy rules in practice", Carnegie-Rochester Conferences Series on Public Policy, vol. 39, $\mathrm{N}^{\circ} 1$, Amsterdam, Elsevier.

West, K. (2006), "Forecast evaluation”, Handbook of Economic Forecasting, G. Elliott, C.W.J. Granger y A. Timmerman (eds.), Amsterdam, Elsevier.

(1996), "Asymptotic inference about predictive ability", Econometrica, vol. 64, $\mathrm{N}^{\circ}$ 5, Nueva York, The Econometric Society.

Yeh, C.C. (2009), "The Simultaneous Evolution of Growth and Inflation", documento presentado en la Conferencia Internacional de Economía Aplicada. 